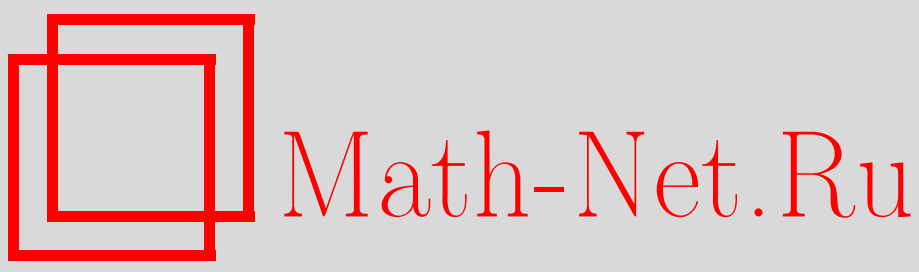

А. Ю. Брудный, А. Л. Онищик, Треугольные когомологии де Рама компактных кэлеровых многообразий, Матем. сб., 2001, том 192, номер 2, 27-56

DOI: https://doi.org/10.4213/sm541

Использование Общероссийского математического портала Math-Net.Ru подразумевает, что вы прочитали и согласны с пользовательским соглашением http://www . mathnet.ru/rus/agreement

Параметры загрузки:

IP: 3.82 .47 .9

26 апреля 2023 г., 03:37:04 
УДК 515.176

\author{
А.Ю. Брудный, А. Л. Онищик
}

\title{
Треугольные когомологии де Рама компактных кэлеровых многообразий
}

\begin{abstract}
Изучаются когомологии де Рама $H_{D R}^{1}(M, G)$ гладкого многообразия $M$ со значениями в группе Ли $G$. По определению это фактормножество множества плоских связностей в тривиальном гладком расслоении $M \times G$ по так называемой калибровочной эквивалентности. Рассматривается случай, когда $M-$ компактное кэлерово многообразие, а $G$ - разрешимая комплексная алгебраическая группа специального класса, который содержит борелевские подгруппы всех комплексных классических групп и, в частности, группу всех треуголњых матриц. В этом случае дается описание множества $H_{D R}^{1}(M, G)$ в терминах когомологий многообразия $M$ со значениями в (абелевых) пучках плоских сечений некоторых плоских расслоений на алгебры Ли со слоем $\mathfrak{g}$ (касательная алгебра группы $G$ ) или, что равносильно, в терминах гармонических форм на $M$, представляющих эти когомологии.

Библиограффия: 11 названий.
\end{abstract}

\section{Введение}

Работа посвящена изучению 1-когомологий де Рама $H_{D R}^{1}(M, G)$ гладкого многообразия $M$ со значениями в группе Ли $G$. Если $G$ неабелева, то $H_{D R}^{1}(M, G)$ не допускает естественной групповой структуры и обычно рассматривается как множество с отмеченной точкой. По определению это фактормножества плоских связностей в тривиальном главном расслоении $M \times G$ относительно так называемой калибровочной эквивалентности, причем отмеченной точкой является класс нулевой связности. В случае, когда $G$ - линейная группа, речь идет о классификации вполне интегрируемых систем линейных дифференциальных уравнений первого порядка на $M$ с точностью до линейных (с функциональными коэффициентами) замен системы неизвестных функций.

Заметим, что множество 1-когомологий де Рама допускает две важные интерпретации. Во-первых, $H_{D R}^{1}(M, G)$ естественным образом вкладьвается в множество 1-когомологий Чеха $H^{1}(M, G)$, причем образ интерпретируется как множество гладко (или топологически) тривиальных плоских главных расслоений с базой $M$ и структурной группой $G$. В случае $G=\mathbb{R}$ это соответствие есть классический изоморфизм де Рама. Во-вторых, любая плоская связность определяет гомоморфизмголономии $\pi_{1}(M) \rightarrow G$, что приводит к инъективному отображению $H_{D R}^{1}(M, G) \rightarrow \operatorname{Hom}\left(\pi_{1}(M), G\right) / \operatorname{Int} G$. Если $G$ стягиваема (например, разрешима

Работа второго автора вьполнена при частичной поддержке Российского фонда фундаментальных исследований (грант № 98-01-00329).

(C) А. Ю. Брудный, А. Л. Онишик 2001 
и односвязна), то любое гладкое главное расслоение со структурной группой $G$ тривиально и обе инъекции являются биекциями.

Мы рассматриваем случай, когда $M$ - компактное кэлерово многообразие, а $G$ - разрешимая комплексная линейная алгебраическая группа специального класса, содержашего борелевские подгруппы всех комплексных классических групп и, в частности, группу $T_{n}(\mathbb{C})$ всех невырожденных треугольных матриц порядка $n$. В этом случае мы даем описание множества $H_{D R}^{1}(M, G)$ в терминах когомологий многообразия $M$ со значениями в (абелевых) пучках плоских сечений некоторых плоских расслоений на алгебры Ли со слоем $\mathfrak{g}$ (алгебра Ли группы $G$ ) или, что равносильно, в терминах гармонических форм на $M$, представляющих эти когомологии. Наш метод основан на использовании множества неабелевых когомологий Дольбо $H^{0,1}(M, G)$ многообразия $M$ со значениями в $G$, которое определяется аналогично множеству когомологий де Рама, и сушественно использует теорию Ходжа на компактном кэлеровом многообразии.

Исследование неабелевых когомологий де Рама и Дольбо имеет достаточно давнюю историю (см., в частности, работы [1]-[4], о связи которых с настоящей работой см. замечание 3.4). Основные результаты настоящей работы были получены в диссертации [5] первого автора в случае, когда $G=T_{n}(\mathbb{C})$. Отметим, что диссертация содержит приложения этих результатов к изучению фундаментальной групшы компактного кэлерова многообразия (см. также [6]).

Работа делится на три параграфа. Первый из них содержит необходимые факты о неабелевых когомологиях де Рама и Дольбо. В частности, очень важна для дальнейшего описанная там техника подкручивания. Доказательства мы опускаем, отсылая читателя к [7], [8]. В $§ 2$ мы излагаем некоторые факты теории Ходжа для плоских векторных расслоений и применяем их к специальному случаю плоских расслоений, структурная группа которых есть группа треугольных матриц с унитарной диагональю. Параграф 3 содержит формулировки и доказательства основных результатов работы. Мы вводим в рассмотрение так называемое свойство Ходжа для разрешимых комплексных алгебраических групп. Лемма 3.1 дает метод для конструкции груп, обладающих этим свойством, а теорема 3.1 утверждает, что этот класс групп включает борелевские подгруппы редуктивных групп, все простые факторы которых являются классическими или имеют тип $E_{6}, E_{7}$. Из того, что $G$ обладает свойством Ходжа, вытекает, в частности, что естественное отображение $\Pi_{0,1}^{*}: H_{D R}^{1}(M, G) \rightarrow H^{0,1}(M, G)$ сюръективно, если $M$ - компактное кэлерово многообразие. Теорема 3.2 описывает так называемые канонические представители классов когомологий де Рама в терминах гармонических форм со значениями в некоторых расслоениях на алгебры Ли. Окончательный результат об описании множества $H_{D R}^{1}(M, G)$ сформулирован в теореме 3.3 . Последний пункт $\S 3$ содержит два дополнительных результата, которые доказываются так же, как теорема 3.3. Теорема 3.4 дает описание подкрученного варианта когомологий де Рама компактного кэлерова многообразия $M$ со значениями в унипотентном радикалегруппы $G$, обладаюшей свойством Ходжа. В теореме 3.5 рассматривается ситуация, когда такая группа $G$ является подгруппой некоторой алгебраической групшы $\widehat{G}$. Мы описываем здесь фактормножество множества плоских связностей $\omega$ на $M \times \widehat{G}$ таких, что $\Pi_{0,1} \omega$ принимает свои значения в $\mathfrak{g}$, относительно калибровочной эквивалентности, определенной группой $G$. 


\section{§1. Когомологии де Рама и Дольбо со значениями в группе Ли}

1.1. В этом пункте описывается нелинейный комплекс, который в классическом абелевом случае совпадает с начальной частью обычного комплекса де Рама гладкого многообразия. Мы следуем работам $[7 ; \S 4$ и $\S 5]$ и [8; $§ 2]$.

Введем сначала некоторые обозначения. Пусть $X$ - топологическое пространство и $\mathscr{F}$ - пучок (не обязательно абелевых) групп на $X$. Тогда определяется групnа 0-когомологий $H^{0}(X, \mathscr{F})$, совпадающая с группой глобальных сечений $\Gamma(X, \mathscr{F})$. Определяется также множество 1-когомологий Чеха $H^{1}(X, \mathscr{F})$, которое в обшем случае не является группой, а лишш множеством с отмеченной точкой. Пусть $\mathfrak{U}$ - открытое покрытие пространства $X$. Обычным образом определяются групшы $p$-коцепей $C^{p}(\mathfrak{U}, \mathscr{F}), p \geqslant 0$, и множество 1-коциклов

$$
Z^{1}(\mathfrak{U}, \mathscr{F})=\left\{z \in C^{1}(\mathfrak{U}, \mathscr{F}): z_{i j} z_{j k}=z_{i k} \quad \text { в } \quad U_{i} \cap U_{j} \cap U_{j} \neq \varnothing\right\}
$$

Имеется действие $\rho$ группы $C^{0}(\mathfrak{U}, \mathscr{F})$ на $C^{1}(\mathfrak{U}, \mathscr{F})$, заданное формулой

$$
(\rho(a)(c))_{i j}=c_{i} z_{i j} c_{j}^{-1}
$$

Подмножество $Z^{1}(\mathfrak{U}, \mathscr{F})$ инвариантно относительно $\rho$. Образуя фактормножество $H^{1}(\mathfrak{U}, \mathscr{F})=Z^{1}(\mathfrak{U}, \mathscr{F}) / \rho\left(C^{0}(\mathfrak{U}, \mathscr{F})\right)$ и переходя к пределу по всем открытым покрытиям $\mathfrak{U}$, получаем множество 1-когомологий $H^{1}(X, \mathscr{F})$. Его отмеченная точка $\varepsilon$ есть класс единичного коцикла $e \in Z^{1}(\mathfrak{U}, \mathscr{F})$.

Пусть теперь $G$ - группа Ли. Для любого гладкого многообразия $M$ обозначим через $\mathscr{F}_{G}$ пучок ростков гладких $G$-значных функций на $M$. В частности, $\mathscr{F}=\mathscr{F}_{\mathbb{R}}$ есть структурный пучок многообразия $M$. Ясно, что $\mathscr{F}_{G}-$ пучок групा. Группа 0-когомологий $H^{0}\left(M, \mathscr{F}_{G}\right)$ - это группа $F_{G}$ глобальных гладких функций $M \rightarrow G$, а множество 1-когомологий $H^{1}\left(M, \mathscr{F}_{G}\right)$ обычно отождествляется с множеством всех гладких главных расслоений с базой $M$ и структурной группой $G$ (рассматриваемых с точностью до изоморфизма). А именно, если $\mathfrak{U}=\left(U_{i}\right)$ - открытое покрытие многообразия $M$, то любой $z \in Z^{1}\left(\mathfrak{U}, \mathscr{F}_{G}\right)$ определяет главное расслоение с функциями перехода $z_{i j}$, которое получается из $M \times G$ путем подкручивания с помощью $z$; это и есть расслоение, соответствующее классу когомологий $\zeta \in H^{1}\left(M, \mathscr{F}_{G}\right)$ коцикла $z$. Единичный элемент $\varepsilon \in H^{1}\left(M, \mathscr{F}_{G}\right)$ соответствует тривиальному расслоению $M \times G$.

Очевидно, постоянньй пучок $G$ на $M$ можно отождествить с подпучком в $\mathscr{F}_{G}$, состоящим из ростков плоских (т.е. локально постоянных) функций. Далее, группа $H^{0}(M, G)$ есть группа всех плоских функций $M \rightarrow G$. Множество $H^{1}(M, G)$ будет интерпретироваться как множество плоских главных расслоений с базой $M$ и структурной группой $G$, т.е. главных расслоений с локально постоянньми функциями перехода, рассматриваемых с точностью до соответствующего изоморфизма.

Пусть $\mathfrak{g}$ - касательная алгебра Ли группы $G$. Рассмотрим обычный $\mathfrak{g}$-значньй комплекс де Рама $\left(A_{\mathfrak{g}}, d\right)$, где $A_{\mathfrak{g}}=\bigoplus_{p \geqslant 0} A_{\mathfrak{g}}^{p}$ - градуированное векторное пространство g-значных гладких форм на $M$. Заметим, что $A_{\mathfrak{g}}$ есть градуированная супералгебра Ли относительно операции $[\cdot, \cdot]$, индуцированной коммутатором в $\mathfrak{g}$. При этом внешний дифференциал $d$ является дифференцированием степени 1. 
Чтобы получить искомый нелинейный комплекс, обозначим через $є \in A_{\mathfrak{g}}^{1}$ каноническую 1-форму на $G$, сопоставляюшую касательному вектору $v$ в точке $g \in G$ вектор $d_{g} r_{g}^{-1}(v) \in \mathfrak{g}$, где $r_{g}: x \mapsto x g$ - правый сдвиг, отвечающий элементу $g$. Рассмотрим тройку групп $R_{G}=\left\{R_{G}^{0}, R_{G}^{1}, R_{G}^{2}\right\}=\left\{F_{G}, A_{\mathfrak{g}}^{1}, A_{\mathfrak{g}}^{2}\right\}$ и определим операторы кограницы $\delta_{0}: R_{G}^{0} \rightarrow R_{G}^{1}$ и $\delta_{1}: R_{G}^{1} \rightarrow R_{G}^{2}$ формулами

$$
\begin{aligned}
\delta_{0}(g) & =g^{*}(\varpi), & g \in F_{G}, \\
\delta_{1}(\alpha) & =d \alpha-\frac{1}{2}[\alpha, \alpha], & \alpha \in A_{\mathfrak{g}}^{1} .
\end{aligned}
$$

Заметим, что группа $R_{G}^{0}=F_{G}$ действует на градуированной алгебре $A_{\mathfrak{g}}$ при помощи автоморфизмов, индуцированных присоединенньм представлением Ad группы $G$. Обозначая это действие через $\mathrm{Ad}$, имеем

$$
d(\operatorname{Ad} g(\alpha))=\operatorname{Ad} g(d \alpha)+\left[\delta_{0}(g), \operatorname{Ad} g(\alpha)\right]
$$

Легко проверить, что $\delta_{0}: F_{G} \rightarrow A_{\mathfrak{g}}^{1}$ есть скрещенный гомоморфизм относительно Ad, т.е.

$$
\delta_{0}(g h)=\delta_{0}(g)+\operatorname{Ad} g\left(\delta_{0}(h)\right) .
$$

Поэтому возникает следующее аффинное действие группы $R_{G}^{0}$ на $R_{G}^{1}=A_{\mathfrak{g}}^{1}$ при помощи так называемых калибровочных преобразований:

$$
\rho(g)(\alpha)=\operatorname{Ad} g(\alpha)+\delta_{0}(g) .
$$

В дальнейшем $G$ будет у нас подгруппой Ли линейной группы $\mathrm{GL}_{m}(\mathbb{R})$. В этом случае операторы кограницы $\delta_{p}$ и действия $\mathrm{Ad}$ и $\rho$ можно записать следуюшим образом:

$$
\begin{aligned}
\delta_{0}(g) & =(d g) g^{-1}, \\
\delta_{1}(\alpha) & =d \alpha-\alpha \wedge \alpha, \\
\operatorname{Ad} g(\alpha) & =g \alpha g^{-1}, \\
\rho(g)(\alpha) & =g \alpha g^{-1}+(d g) g^{-1} .
\end{aligned}
$$

Используя (1.3), легко проверить следуюшее соотношение:

$$
\delta_{1} \circ \rho(g)=\operatorname{Ad} g \circ \delta_{1}
$$

Это значит, что тройка $R_{G}$ вместе с отображениями $\delta_{p}, p=0,1$, и действиями $\mathrm{Ad}$ и $\rho$ является неабелевым коцепным комплексом в смысле [7], [8]. Он называется комплексом де Рама со значениями в $G$.

Определим множества коциклов

$$
Z^{p}\left(R_{G}\right)=\operatorname{Ker} \delta_{p}=\delta_{p}^{-1}(0), \quad p=0,1
$$


Тогда $Z^{0}\left(R_{G}\right)$ - подгруппа в $F_{G}$. Далее, подмножество $Z^{1}\left(R_{G}\right)$ инвариантно относительно $\rho$ в силу (1.6). Определим когомологии де Рама многообразия $M$ со значениями в $G$ (или когомологии комплекса $R_{G}$ ) формулами

$$
\begin{aligned}
& H_{D R}^{0}(M, G)=H^{0}\left(R_{G}\right)=Z^{0}\left(R_{G}\right) \\
& H_{D R}^{1}(M, G)=H^{1}\left(R_{G}\right)=Z^{1}\left(R_{G}\right) / \rho\left(F_{G}\right) .
\end{aligned}
$$

Имеем $H_{D R}^{0}(M, G)=H^{0}(M, G)$. Если группа $G$ неабелева, то множество когомологий $H_{D R}^{1}(M, G)$ не допускает естественной групповой структуры. Мы рассматриваем его как множество с отмеченной точкой $\varepsilon=\rho\left(F_{G}\right)(0)$. Класс когомологий коцикла $\omega \in Z^{1}\left(R_{G}\right)$ будет обозначаться через $[\omega]$.

Между 1-когомологиями де Рама и Чеха имеется следуюшее соотношение (см. $[7 ; \S 5])$.

ПРЕДЛОЖЕНИЕ 1.1. Имеем следующую точную последовательность множсеств с отмеченными точками:

$$
e \rightarrow H_{D R}^{1}(M, G) \stackrel{\mu}{\rightarrow} H^{1}(M, G) \stackrel{i^{*}}{\rightarrow} H^{1}\left(M, \mathscr{F}_{G}\right)
$$

Здесь $i^{*}$ определяется вложением $i: G \rightarrow \mathscr{F}_{G}$, а $\mu$ действует следующим образом. Для любого коиикла $\omega \in Z^{1}\left(R_{G}\right)$ выберем такое открытое покрытие $\mathfrak{U}=\left(U_{i}\right)$ многообразия $M$, что $\omega=\delta_{0}\left(c_{i}\right)$ в кажсдом $U_{i}$ для некоторых гладких $c_{i}: U_{i} \rightarrow G$. Тогда $z_{i j}=c_{i}^{-1} c_{j}$ составляют коцикл $z=\left(z_{i j}\right) \in Z^{1}(\mathfrak{U}, G)$ и нотображсает $[\omega]$ в класс когомологий коцикла $z$.

Точность последовательности (1.7) означает, что $\mu$ биективно отображает множество $H_{D R}^{1}(M, G)$ на подмножество в $H^{1}(M, G)$, состоящее из плоских расслоений, тривиальных как гладкие расслоения. При этом $\mu([0])=\varepsilon$.

Если $G$ абелева, то (1.7) - точная последовательность групп и их гомоморфизмов. В частности, для $G=\mathbb{R}$ комплекс $R_{G}$ есть часть классического комплекса де Рама и $\mu: H_{D R}^{1}(M, \mathbb{R}) \rightarrow H^{1}(M, \mathbb{R})$ есть изоморфизм де Рама для 1-когомологий.

Аналогичньм образом определяется неабелев аналог классического комплекса Дольбо (здесь мы следуем $[7 ; \S 6])$.

Пусть $G$ - комплексная группа Ли. Для любого комплексного многообразия $M$ обозначим через $\mathscr{O}_{G}$ пучок ростков $G$-значных голоморфных функций на $M$. В частности, $\mathscr{O}=\mathscr{O}_{\mathbb{C}}$ есть структурньй пучок многообразия $M$. Тогда $H^{0}\left(M, \mathscr{O}_{G}\right)$ - это группа всех $G$-значных голоморфных функций на $M$, a $H^{1}\left(M, \mathscr{O}_{G}\right)$ можно интерпретировать как множество всех голоморфных главных расслоений с базой $M$ и структурной группой $G$, которые рассматриваются с точностью до голоморфных изоморфизмов, оставляющих на месте каждую точку из $M$. Единичный класс $\varepsilon$ соответствует тривиальному расслоению $M \times G$.

В этом случае $A_{\mathfrak{g}}=\bigoplus_{p, q \geqslant 0} A_{\mathfrak{g}}^{p, q}$ есть биградуированная комплексная супералгебра Ли, где $A_{\mathfrak{g}}^{p, q}$ - пространство форм типа $(p, q)$. Пусть $\Pi_{p, q}: A_{\mathfrak{g}}^{p+q} \rightarrow A_{\mathfrak{g}}^{p, q}$ естественная проекция. Как обычно, обозначим

$$
\partial \alpha=\Pi_{p+1, q} d \alpha, \quad \bar{\partial} \alpha=\Pi_{p, q+1} d \alpha, \quad \alpha \in A_{\mathfrak{g}}^{p, q}
$$


Тогда

$$
d=\partial+\bar{\partial}, \quad \partial^{2}=\bar{\partial}^{2}=[\partial, \bar{\partial}]=0 .
$$

Здесь $\partial$ и $\bar{\partial}$ являются операторами дифференцирования бистепени $(1,0)$ и $(0,1)$ соответственно. Мы получили биградуированные комплексы Дольбо $\left(A_{\mathfrak{g}}, \bar{\partial}\right)$ и $\left(A_{\mathfrak{g}}, \partial\right)$. Обозначая через $\Omega_{\mathfrak{g}}^{p}$ и $\bar{\Omega}_{\mathfrak{g}}^{p}$ соответственно пучки голоморфных и антиголоморфных $p$-форм на $M$, имеем классические изоморфизмы Дольбо

$$
\begin{aligned}
& H^{p, q}(M, \mathfrak{g}) \stackrel{\text { def }}{=} H^{q}\left(A_{\mathfrak{g}}^{p, *}, \bar{\partial}\right) \simeq H^{q}\left(M, \Omega_{\mathfrak{g}}^{p}\right), \\
& \bar{H}^{p, q}(M, \mathfrak{g}) \stackrel{\text { def }}{=} H^{p}\left(A_{\mathfrak{g}}^{*, q}, \partial\right) \simeq H^{p}\left(M, \bar{\Omega}_{\mathfrak{g}}^{q}\right) .
\end{aligned}
$$

Рассмотрим теперь тройку групा $\bar{R}_{G}=\left\{\bar{R}_{G}^{0}, \bar{R}_{G}^{1}, \bar{R}_{G}^{2}\right\}=\left\{F_{G}, A_{\mathfrak{g}}^{0,1}, A_{\mathfrak{g}}^{0,2}\right\}$ и определим операторы кограницы $\bar{\delta}_{0}: \bar{R}_{G}^{0} \rightarrow \bar{R}_{G}^{1}, \bar{\delta}_{1}: \bar{R}_{G}^{1} \rightarrow \bar{R}_{G}^{2}$ и действие $\bar{\rho}$ группы $\bar{R}_{G}^{0}$ на $\bar{R}_{G}^{1}$ формулами

$$
\begin{aligned}
\bar{\delta}_{0}(g) & =\Pi_{0,1} g^{*}(\varpi), \\
\bar{\delta}_{1}(\alpha) & =\bar{\partial} \alpha-\frac{1}{2}[\alpha, \alpha], \\
\bar{\rho}(g)(\alpha) & =\operatorname{Ad} g(\alpha)+\bar{\delta}_{0}(g) .
\end{aligned}
$$

Мы получаем неабелев коцепной комплекс в смысле [7], [8], называемый комплексом Дольбо со значениями в $G$. Если $G$-подгруппа Ли в $\mathrm{GL}_{m}(\mathbb{C})$, то

$$
\begin{aligned}
\bar{\delta}_{0}(g) & =(\bar{\partial} g) g^{-1} \\
\bar{\delta}_{1}(\alpha) & =\bar{\partial} \alpha-\alpha \wedge \alpha, \\
\bar{\rho}(g)(\alpha) & =g \alpha g^{-1}+(\bar{\partial} g) g^{-1} .
\end{aligned}
$$

Множества коциклов этого комплекса определяются формулами

$$
Z^{p}\left(\bar{R}_{G}\right)=\operatorname{Ker} \bar{\delta}_{p}=\bar{\delta}_{p}^{-1}(0), \quad p=0,1
$$

Тогда $Z^{0}\left(\bar{R}_{G}\right)$ есть подгруппа в $F_{G}$, а подмножество $Z^{1}\left(\bar{R}_{G}\right)$ инвариантно относительно $\bar{\rho}$. Определим когомологии Дольбо со значениями в $G$ (или когомологии комплекса $\bar{R}_{G}$ ) формулами

$$
\begin{aligned}
H^{0}\left(\bar{R}_{G}\right) & =Z^{0}\left(\bar{R}_{G}\right), \\
H^{0,1}(M, G) & =H^{1}\left(\bar{R}_{G}\right)=Z^{1}\left(\bar{R}_{G}\right) / \bar{\rho}\left(F_{G}\right) .
\end{aligned}
$$

Имеем следуюшее соотношение между 1-когомологиями Дольбо и Чеха (см. $[7 ; \S 6])$. 
ПРЕДЛОЖЕНИЕ 1.2. Имеем следующую точную последовательность множеств с отмеченными точками:

$$
e \rightarrow H^{0,1}(M, G) \stackrel{\bar{\mu}}{\rightarrow} H^{1}\left(M, \mathscr{O}_{G}\right) \stackrel{i^{*}}{\rightarrow} H^{1}\left(M, \mathscr{F}_{G}\right)
$$

Здесь $i^{*}$ определяется вложсением $i: \mathscr{O}_{G} \rightarrow \mathscr{F}_{G}$, а $\bar{\mu}$ действует следующим образом. Для любого кочикла $\omega \in Z^{1}\left(\bar{R}_{G}\right)$ выберем такое открытое покрытие $\mathfrak{U}=\left(U_{i}\right)$ многообразия $M$, что $\omega=\bar{\delta}_{0}\left(c_{i}\right)$ в каждом $U_{i}$ для некоторьх гладких $c_{i}: U_{i} \rightarrow G$. Тогда $z_{i j}=c_{i}^{-1} c_{j}$ составляют кочикл $z=\left(z_{i j}\right) \in Z^{1}\left(\mathfrak{U}, \mathscr{O}_{G}\right)$ u $\bar{\mu}$ отображсает класс когомологий Дольбо $[\omega]$ формьи $\omega$ в класс когомологий кочикла z.

Это означает, что $\bar{\mu}$ биективно отображает множество $H^{0,1}(M, G)$ на подмножество в $H^{1}\left(M, \mathscr{O}_{G}\right)$, состоящее из голоморфных расслоений, тривиальных как гладкие расслоения. При этом $\bar{\mu}([0])=\varepsilon$.

Если $G$ абелева, то (1.10) - точная последовательность групп и их гомоморфизмов. В частности, для $G=\mathbb{C}$ комплекс $\bar{R}_{G}$ есть часть классического комплекса Дольбо и $\bar{\mu}: H^{0,1}(M, \mathbb{C}) \rightarrow H^{1}(M, \mathscr{O})$ есть изоморфизм Дольбо для $(0,1)$-когомологий.

Конструкции комплексов де Рама и Дольбо определяют ковариантные функторы $G \mapsto R_{G}$ и $G \mapsto \bar{R}_{G}$ из категории вешественных (соответственно комплексных) групп Ли в категорию коцепных комплексов (см. [8]). В частности, любому гладкому гомоморфизму групп Ли $f: G \rightarrow Q$ отвечают гомоморфизм групп $f^{*}: H_{D R}^{0}(M, G) \rightarrow H_{D R}^{0}(M, Q)$ и гомоморфизм множеств с отмеченными точками $f^{*}: H_{D R}^{1}(M, G) \rightarrow H_{D R}^{1}(M, Q)$. Аналогично, любому голоморфному гомоморфизму комплексных групп Ли $f: G \rightarrow Q$ отвечает гомоморфизм множеств с отмеченными точками $f^{*}: H^{0,1}(M, G) \rightarrow H^{0,1}(M, Q)$.

1.2. Если группа $G$ неабелева, то $i^{*}$ в $(1.7)$ и (1.10) не является гомоморфизмом групп, и потому слои этого отображения нельзя описать при помощи его ядра. Описание этих слоев дается с помошью подкрученных вариантов нелинейных комплексов де Рама и Дольбо.

Пусть $\mathscr{S}$ - пучок групп на $X$ и $\mathscr{A} u t \mathscr{S}$ - пучок ростков автоморфизмов этого пучка. Выберем открытое покрытие $\mathfrak{U}=\left(U_{i}\right)$ пространства $X$ и фиксируем коцикл $z \in Z^{1}(\mathfrak{U}, \mathscr{A} u t \mathscr{S})$. Тогда мы можем подкрутить $\mathscr{S}$ при помоши $z$, получив при этом новьй пучок групп $\mathscr{S}^{z}$ на $X$. Для этого мы склеим между собой любые два пучка $\mathscr{S} \mid U_{i}$ и $\mathscr{S} \mid U_{j}$ над $U_{i} \cap U_{j} \neq \varnothing$, отождествляя $a_{i} \in\left(\mathscr{S} \mid U_{i}\right)_{x}$ с $a_{j} \in\left(\mathscr{S} \mid U_{j}\right)_{x}$, $x \in U_{i} \cap U_{j}$, при условии $a_{i}=z_{i j}\left(a_{j}\right)$. Подкрученныи пучок $\mathscr{S}^{z}$ зависит с точностью до изоморфизмов только от класса когомологий $\zeta \in H^{1}(X, \mathscr{A} u t \mathscr{S})$, определяемого коциклом $z$. Например, если $z$ когомологичен коциклу $e$, т.е. $z_{i j}=c_{i} c_{j}^{-1}$ для некоторой 0-коцепи $c \in C^{0}(\mathfrak{U}, \mathscr{A} u t \mathscr{S})$, то из равенства $a_{i}=z_{i j}\left(a_{j}\right)$ следует, что $c_{i}^{-1}\left(a_{i}\right)=c_{j}^{-1}\left(a_{j}\right)$. Значит, соответствие $\left(a_{i}\right) \mapsto\left(c_{i}^{-1}\left(a_{i}\right)\right)$ есть изоморфизм пучка $\mathscr{S}^{z}$ на $\mathscr{S}$. Заметим также, что любое сечение $s \in \Gamma\left(U, \mathscr{S}^{z}\right)$ задается 0-коцепю $\left(s_{i}\right)$, где $s_{i} \in \Gamma\left(U \cap U_{i}, \mathscr{S}\right)$, удовлетворяющей условиям $s_{i}=z_{i j}\left(s_{j}\right)$ над $U \cap U_{i} \cap U_{j} \neq \varnothing$. 
Та же конструкция применима к пучкам колец, векторных пространств и т.д. Аналогичным образом производится подкручивание расслоения при помощи коцикла со значениями в пучке его автоморфизмов. Рассмотрим некоторые частные случаи этой конструкции, важные для дальнейшего.

Пусть $G$ - подгруппа Ли в $\mathrm{GL}_{m}(\mathbb{R})$ и $M$-гладкое многообразие. Для некоторого открытого покрытия $\mathfrak{U}=\left(U_{i}\right)$ многообразия $M$ фиксируем коцикл $z \in Z^{1}(\mathfrak{U}, G)$. Очевидно, $z$ определяет плоское векторное расслоение $\mathbf{V}=\mathbf{V}(z)$ ранга $m$ над $M$ со структурной группой $G$, а именно расслоение со слоем $\mathbb{R}^{m}$ и плоскими функциями перехода $z_{i j}$, которое получается из $M \times \mathbb{R}^{m}$ путем подкручивания при помощи коцикла $z$. Обозначим через $A_{\mathbf{V}}^{p}, p \geqslant 0$, пространство гладких $\mathbf{V}$-значных $p$-форм. Такая форма $\alpha$ задается по определению набором $\mathbb{R}^{m}$-значных $p$-форм $\alpha_{i}$ в $U_{i}$, удовлетворяюшим условиям $\alpha_{i}=z_{i j}\left(\alpha_{j}\right)$ над $U_{i} \cap U_{j} \neq \varnothing$, т.е. является сечением пучка $\mathbb{R}^{m}$-значных $p$-форм на $M$, подкрученного при помощи коцикла $z$. Очевидно, формула $(d \alpha)_{i}=d \alpha_{i}$ корректно определяет оператор $d: A_{\mathbf{V}}^{p} \rightarrow A_{\mathbf{V}}^{p+1}$, причем пара $\left(A_{\mathbf{V}}, d\right)$, где $A_{\mathbf{V}}=\bigoplus_{p \geqslant 0} A_{\mathbf{V}}^{p}$, является коцепным комплексом. Имеем $H^{p}\left(A_{\mathbf{V}}, d\right) \simeq H^{p}(M, \mathscr{C} \mathbf{V})$, где $\mathscr{C} \mathbf{V}-$ пучок ростков плоских сечений расслоения $\mathbf{V}$.

Пусть теперь $G$ - подгруппа Ли в $\mathrm{GL}_{m}(\mathbb{C}), M$ - комплексное многообразие и $z \in Z^{1}\left(\mathfrak{U}, \mathscr{O}_{G L_{m}(\mathbb{C})}\right)$, причем значения функций $z_{i j}$ принадлежат $G$. Тогда определяется голоморфное векторное расслоение $\mathbf{V}=\mathbf{V}(z)$ ранга $m$ над $M$ со структурной группой $G$. С ним связан биградуированньй комплекс Дольбо $\left(A_{\mathbf{V}}, \bar{\partial}\right)$, причем имеют место изоморфизмы Дольбо

$$
H^{p, q}(M, \mathbf{V}) \stackrel{\text { def }}{=} H^{q}\left(A_{\mathbf{V}}^{p, *}, \bar{\partial}\right) \simeq H^{q}\left(M, \Omega_{\mathbf{V}}^{p}\right)
$$

где $\Omega_{\mathbf{V}}^{p}$ - пучок $\mathbf{V}$-значных голоморфных $p$-форм на $M$. В дальнейшем нас будет интересовать случай, когда $z \in Z^{1}(\mathfrak{U}, G)$, т.е. когда расслоение $\mathbf{V}$ является плоским. В этом случае определен также биградуированньй комплекс $\left(A_{\mathbf{V}}, \partial\right)$. При этом

$$
\bar{H}^{p, q}(M, \mathbf{V}) \stackrel{\text { def }}{=} H^{p}\left(A_{\mathbf{V}}^{*, q}, \partial\right) \simeq H^{p}\left(M, \bar{\Omega}_{\mathbf{V}}^{q}\right),
$$

где $\bar{\Omega}_{\mathbf{V}}^{p}$ - пучок $\mathbf{V}$-значных антиголоморфных $p$-форм на $M$.

Чтобы определить подкрученный комплекс де Рама со значениями в групп Ли $G$, предположим, что задан коцикл $\mathfrak{z} \in Z^{1}(\mathfrak{U}$, Aut $G)$, где Aut $G$ - группа автоморфизмов группы Ли $G$. Рассмотрим подкрученньй пучок групп $G^{\mathfrak{z}}$. Его можно реализовать как пучок $\mathscr{C}_{E}$ плоских сечений плоского расслоения на группы $E$, которое получается из $M \times G$ путем подкручивания с помощью коцикла $\mathfrak{z}$. Любой автоморфизм группы Ли $G$ индуцирует автоморфизм пучка $\mathscr{F}_{G}$, и поэтому мы получаем подкрученный пучок $\left(\mathscr{F}_{G}\right)^{\mathfrak{z}}$, совпадающий с пучком $\mathscr{F}_{E}$ гладких сечений расслоения $E$. Мы имеем также коцикл $d \mathfrak{z}$ автоморфизмов алгебры Ли $\mathfrak{g}$, приводящий к плоскому расслоению на алгебры Ли $\mathfrak{e}$ со слоем $\mathfrak{g}$, которое получается из $M \times \mathfrak{g}$ путем подкручивания. Рассмотрим комплекс де Рама $\left(A_{\mathfrak{e}}, d\right)$. Подкрученный нелинейный комплекс определяется как тройка $R_{E}=\left\{R_{E}^{0}, R_{E}^{1}, R_{E}^{2}\right\}$, где $R_{E}^{0}=F_{E}=\Gamma(M, \mathscr{F} E), R_{E}^{p}=A_{\mathfrak{e}}^{p}, p=1,2$. Кограничные операторы и действия 
группы $F_{E}$ корректно определяются формулами

$$
\begin{aligned}
& \left(\delta_{0}(f)\right)_{i}=\left(\delta_{0}\left(f_{i}\right)\right) \quad \text { для } f=\left(f_{i}\right) \in F_{E}, \\
& \left(\delta_{1}(\alpha)\right)_{i}=\left(\delta_{1}\left(\alpha_{i}\right)\right) \quad \text { для } \alpha=\left(\alpha_{i}\right) \in A_{\mathfrak{e}}^{1}, \\
& (\operatorname{Ad} f(\alpha))_{i}=\left(\operatorname{Ad} f_{i}(\alpha)_{i}\right) \quad \text { для } \alpha=\left(\alpha_{i}\right) \in A_{\mathfrak{e}}^{p} \text {, } \\
& (\rho(f)(\alpha))_{i}=\left(\rho\left(f_{i}\right)(\alpha)_{i}\right) \quad \text { для } \alpha=\left(\alpha_{i}\right) \in A_{\mathfrak{e}}^{1} .
\end{aligned}
$$

Как и вьше, мы получаем неабелев коцепной комплекс. Определяются группа 0-когомологий $H_{D R}^{0}(M, E)=H^{0}\left(R_{E}\right)=Z^{0}\left(R_{E}\right)$ и множество 1-когомологий $H_{D R}^{1}(M, E)=H^{1}\left(R_{E}\right)=Z^{1}\left(R_{E}\right) / \rho\left(F_{E}\right)$. Группа $H_{D R}^{0}(M, E)$ совпадает с группой $H^{0}\left(M, \mathscr{C}_{E}\right)$ плоских сечений расслоения $E$. Обобщая $(1.7)$, получаем следующую точную последовательность множеств с отмеченными точками:

$$
e \rightarrow H_{D R}^{1}(M, E) \stackrel{\mu}{\rightarrow} H^{1}\left(M, \mathscr{C}_{E}\right) \stackrel{i^{*}}{\rightarrow} H^{1}\left(M, \mathscr{F}_{G}\right)
$$

Будем обозначать через Int $h$ внутренний автоморфизм $x \mapsto h x h^{-1}$ группы $H$, определенный элементом $h \in H$. Предположим, что $\mathfrak{z}=\operatorname{Int} z$, т.е. $\mathfrak{z} i j=\operatorname{Int} z_{i j}$, где $z=\left(z_{i j}\right) \in Z^{1}(\mathfrak{U}, G)$. Обозначим через $P$ плоское главное расслоение со структурной группой $G$ над $M$, соответствующее коциклу $z$. Тогда $E=\operatorname{Int} P$ есть расслоение на группы, ассоциированное с $P$ при помощи действия Int группы $G$ на себе внутренними автоморфизмами, а $\mathfrak{e}=\operatorname{Ad} P$ - расслоение на алгебры Ли, ассоциированное с $P$ при помощи присоединенного представления Аd группы $G$ на g. Векторное пространство $R_{\text {Int } P}^{1}=A_{\text {Ad } P}^{1}$ можно интерпретировать как пространство связностей в $P$ (рассматриваемом как гладкое главное расслоение), a $\delta_{1}(\alpha) \in R_{\text {Int } P}^{2}=A_{\text {Ad } P}^{2}-$ как кривизну связности $\alpha$, так что $Z^{1}\left(R_{\text {Int } P}\right)$ отождествляется с множеством связностей нулевой кривизны (или плоских связностей). Далее, $R_{\text {Int } P}^{0}=F_{\text {Int } P}$ отождествляется с группой гладких автоморфизмов главного расслоения $P$ (индуцирующих тождественное преобразование базы $M$ ), а $\rho$ - с действием этой групшы на $A_{\text {Ad } P}^{1}$ при помощи калибровочных преобразований. По определению $H^{1}\left(R_{\operatorname{Int} P}\right)=H_{D R}^{1}(M, \operatorname{Int} P)$ есть фактормножество множества $Z^{1}\left(R_{\text {Int } P}\right)$ по этому действию, а $H^{0}\left(R_{\text {Int } P}\right)=H^{0}\left(M, \mathscr{C}_{\text {Int } P}\right)$ есть группа плоских автоморфизмов расслоения $P$.

Предположим, что $G \subset \mathrm{GL}_{n}(\mathbb{C})$ - подгруппа Ли в $\mathrm{GL}_{n}(\mathbb{R})$ или $\mathrm{GL}_{n}(\mathbb{C})$. Тогда $E=\operatorname{Int} P$ можно рассматривать как подрасслоение плоского векторного расслоения End V $(z)$, соответствуюшего коциклу Int $z=\operatorname{Ad} z$. Группа $F_{E}$ совпадает с некоторой подгруппой групшы Aut $\mathbf{V}(z)$ автоморфизмов векторного расслоения $\mathbf{V}(z)$.

Рассмотрим теперь специальньй случай, когда $P$ тривиально как гладкое расслоение, т.е. когда класс когомологий $\zeta$ коцикла $z$ лежит в $\operatorname{Im} \mu$ (см. предложение 1.1). Мы можем считать, что $z_{i j}=c_{i}^{-1} c_{j}$, где $c_{i} \in \Gamma\left(U_{i}, \mathscr{F}_{G}\right)$. Тогда $\gamma=\delta_{0}\left(c_{i}\right)$ - это корректно определенная форма из $Z^{1}\left(R_{G}\right)$, причем $[\gamma]$ удовлетворяет условию $\mu([\gamma])=\zeta$. Коцеп $\left(c_{i}\right)$ определяет изоморфизм групп $t: R_{E}^{0} \rightarrow R_{G}^{0}=F_{G}$, заданньй формулой

$$
t\left(\left(g_{i}\right)\right)=c_{i} g_{i} c_{i}^{-1} \text { в } U_{i}
$$


Аналогично, имеем изоморфизм градуированных супералгебр Ли $\tau: A_{\mathfrak{e}} \rightarrow A_{\mathfrak{g}}$, заданньй на формах степени $p$ формулой

$$
\tau_{p}\left(\left(\alpha_{i}\right)\right)=\operatorname{Ad} c_{i}\left(\alpha_{i}\right) \text { в } U_{i}, \quad\left(\alpha_{i}\right) \in A_{\mathfrak{e}}^{p} .
$$

Он не является изоморфизмом комплексов де Рама. Точнее, из (1.3) видно, что

$$
\tau \circ d=(d-\operatorname{ad} \gamma) \circ \tau
$$

где $\operatorname{ad} \gamma: \alpha \mapsto[\gamma, \alpha]-$ присоединенньй оператор в $A_{\mathfrak{g}}$, связанный с $\gamma$. Зададим теперь отображение $r_{\gamma}: A_{\mathfrak{e}}^{1} \rightarrow A_{\mathfrak{g}}^{1}$ формулой

$$
r_{\gamma}\left(\left(\alpha_{i}\right)\right)=\tau_{1}\left(\alpha_{i}\right)+\gamma=\operatorname{Ad} c_{i}\left(\alpha_{i}\right)+\gamma=\rho\left(c_{i}\right)\left(\alpha_{i}\right) .
$$

Очевидно, это корректно определенное биективное аффинное отображение.

ПреДЛОЖЕНИЕ 1.3. Пусть Р тривиально как гладкое расслоение. Тогда в обозначениях, введенных в (1.15), (1.16), (1.17), справедливы следующие соотношения:

$$
\begin{aligned}
& \delta_{1} \circ r_{\gamma}=\tau_{2} \circ \delta_{1}, \\
& r_{\gamma} \circ \rho(g)=\rho(t(g)) \circ r_{\gamma}, \quad g \in F_{E} .
\end{aligned}
$$

Таким образом, $r_{\gamma}$ отображает $Z^{1}\left(R_{E}\right)$ на $Z^{1}\left(R_{G}\right)$ и индуцирует биекцию $r_{\gamma}^{*}: H_{D R}^{1}(M, E) \rightarrow H_{D R}^{1}(M, G)$, переводящую $\varepsilon$ в $[\gamma]$.

Доказательство - непосредственная проверка. Это утверждение следует также из предложения 1.5 работы [8].

Определим теперь подкрученный вариант комплекса Дольбо. Пусть $M-$ комплексное многообразие и $G$ - комплексная группа Ли. Обозначим через $\operatorname{Aut}_{h} G$ группу голоморфных автоморфизмов группы $G$ и выберем $\mathfrak{z} \in Z^{1}\left(\mathfrak{U}, \mathscr{F}_{\mathrm{Aut}_{h}} G\right)$. Очевидно, пучок групп $\mathscr{O}_{\mathrm{Aut}_{h}}$ g действует на $\mathscr{O}_{G}$ и $\mathscr{F}_{G}$. Применяя подкручивание при помощи коцикла $\mathfrak{z}$, получаем голоморфное расслоение на группы $E$, а также пучки $\mathscr{O}_{E}=\left(\mathscr{O}_{G}\right)^{\mathfrak{z}}$ и $\mathscr{F}_{E}=\left(\mathscr{F}_{G}\right)^{\mathfrak{z}}$ соответственно голоморфных и гладких сечений расслоения $E$. Коцикл $d \mathfrak{z}$ приводит к голоморфному расслоению $\mathfrak{e}$ на алгебры Ли со слоем $\mathfrak{g}$.

Соответствующий подкрученный вариант комплекса Дольбо $\bar{R}_{G}$ имеет вид $\bar{R}_{E}=\left\{\bar{R}_{E}^{0}, \bar{R}_{E}^{1}, \bar{R}_{E}^{2}\right\}$, где $\bar{R}_{E}^{0}=F_{E}, \bar{R}_{E}^{p}=A_{\mathfrak{e}}^{0, p}, p=1,2$. Операторы кограницы и действия группы $F_{E}$ определяются формулами, аналогичными (1.13). Группа 0-когомологий этого комплекса - это группа Г $\left(M, \mathscr{O}_{E}\right)$ всех голоморфных сечений расслоения $E$; множество 1-когомологий обозначается через $H^{0,1}(M, E)$. Рассмотрим случай, когда $\mathfrak{z}=\operatorname{Int} z$, где $z=\left(z_{i j}\right) \in Z^{1}\left(\mathfrak{U}, \mathscr{O}_{G}\right)$. Обозначим через $P$ голоморфное главное расслоение над $M$, соответствующее $z$. Тогда $E=\operatorname{Int} P$ есть ассоциированное с $P$ расслоение на групшы, а $\mathfrak{e}=\operatorname{Ad} P$ - ассоциированное расслоение на алгебры Ли.

Пусть теперь $P$ тривиально как гладкое расслоение, т.е. $z_{i j}=c_{i}^{-1} c_{j}$, где $c_{i} \in$ $\Gamma\left(U_{i}, \mathscr{F}_{G}\right)$. Тогда $\gamma=\bar{\delta}_{0}\left(c_{i}\right)$ - корректно определенная форма из $Z^{1}\left(\bar{R}_{G}\right)$, причем класс $[\gamma]$ удовлетворяет условию $\bar{\mu}([\gamma])=\zeta$, где $\zeta \in H^{1}\left(M, \mathscr{O}_{G}\right)$ - класс 
когомологий коцикла $z$. Как и в плоском случае, мы имеем изоморфизм групп $t: F_{E} \rightarrow F_{G}$, заданный формулой (1.15), и изоморфизм биградуированных супералгебр Ли $\tau: A_{\mathfrak{e}} \rightarrow A_{\mathfrak{g}}$, заданньй формулой (1.16). При этом

$$
\tau \circ \bar{\partial}=(\bar{\partial}-\operatorname{ad} \gamma) \circ \tau
$$

Определим также отображение $\bar{r}_{\gamma}: A_{\mathfrak{e}}^{0,1} \rightarrow A_{\mathfrak{g}}^{0,1}$ формулой

$$
\bar{r}_{\gamma}\left(\left(\alpha_{i}\right)\right)=\tau_{1}\left(\alpha_{i}\right)+\gamma=\operatorname{Ad} c_{i}\left(\alpha_{i}\right)+\gamma=\bar{\rho}\left(c_{i}\right)\left(\alpha_{i}\right)
$$

Легко проверяется следуюшее утверждение.

ПРЕДЛОЖЕНИЕ 1.4. Пусть $P$ тривиально как гладкое расслоение. Тогда имеют место следующие соотношения:

$$
\begin{aligned}
\bar{\delta}_{1} \circ \bar{r}_{\gamma} & =\tau_{2} \circ \bar{\delta}_{1}, \\
\bar{r}_{\gamma} \circ \bar{\rho}(g) & =\bar{\rho}(t(g)) \circ \bar{r}_{\gamma}, \quad g \in \bar{R}_{E}^{0} .
\end{aligned}
$$

Таким образом, $\bar{r}_{\gamma}$ отображсает $Z^{1}\left(\bar{R}_{E}\right)$ на $Z^{1}\left(\bar{R}_{G}\right)$ и индуцирует биекцию $\bar{r}_{\gamma}^{*}: H^{0,1}(M, E) \rightarrow H^{0,1}(M, G)$, переводящую $\varepsilon$ в $[\gamma]$.

В дальнейшем мы обычно будем рассматривать случай, когда $E$ плоско, т.е. ког да соответствующий коцикл z принадлежит $Z^{1}\left(\mathfrak{U}, \operatorname{Aut}_{h} G\right)$.

1.3. Между комплексами де Рама и Дольбо со значениями в одной и той же комплексной групе Ли $G$ имеется важное соотношение. Оно возникает благодаря наличию естественного вложения пучков $\iota_{G}: G \rightarrow \mathscr{O}_{G}$. Легко проверить, что тройка $\left\{\mathrm{id}, \Pi_{0,1}, \Pi_{0,2}\right\}$ является гомоморфизмом комплекса де Рама в комплекс Дольбо. Это приводит к следуюшей коммутативной диаграмме:

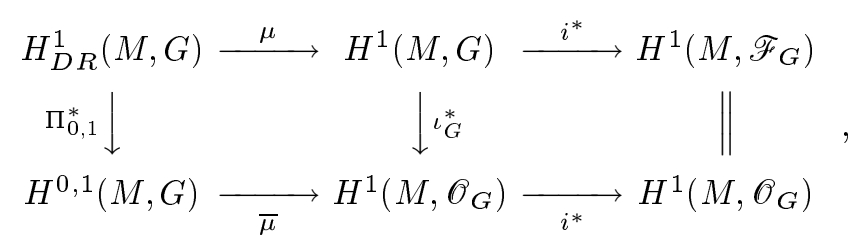

где $\Pi_{0,1}^{*}$ индуцируется проекцией $\Pi_{0,1}$.

В более общей ситуации предположим, что мы имеем плоское расслоение на группы $E$, соответствуюшее коциклу $\mathfrak{z} \in Z^{1}\left(\mathfrak{U}, \mathrm{Aut}_{h} G\right)$. Рассматривая $E$ как голоморфное расслоение на группы Ли, получаем коммутативную диаграмму

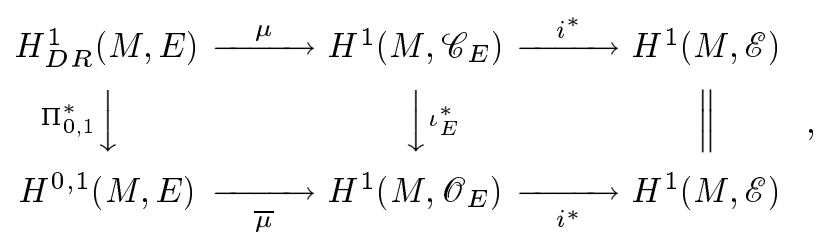

где отображение $\Pi_{0,1}^{*}$ индуцируется проекцией $\Pi_{0,1}$, a $\iota_{E}^{*}-$ естественным вложением $\iota_{E}: \mathscr{C}_{E} \rightarrow \mathscr{O}_{E}$. 
Заметим также, что для любого $\gamma \in Z^{1}\left(R_{G}\right)$ следующая диаграмма является коммутативной:

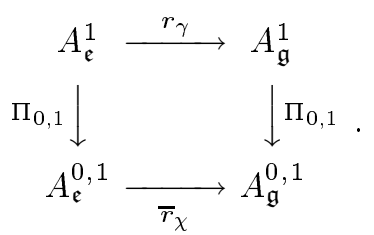

Здесь $\mathfrak{e}$ - плоское расслоение на алгебры Ли, связанное с формой $\gamma$, и $\chi=\Pi_{0,1} \gamma$.

Рассмотрим теперь пример абелевой комплексной группы Ли $\mathrm{GL}_{1}(\mathbb{C})=\mathbb{C}^{\times}$, который будет полезен в дальнейшем. Заметим, что $\mathrm{GL}_{1}(\mathbb{C})$ содержит компактную подгруппу $\mathrm{U}_{1}=\{c \in \mathbb{C}:|c|=1\}$.

ПримеР 1.1. Вычислим групшы $H_{D R}^{1}\left(M, \mathbb{C}^{\times}\right), H^{0,1}\left(M, \mathbb{C}^{\times}\right)$и $H_{D R}^{1}\left(M, \mathrm{U}_{1}\right)$. Рассмотрим гомоморфизм $\operatorname{Exp:~} \mathbb{C} \rightarrow \mathbb{C}^{\times}$, заданный формулой $\operatorname{Exp} c=\exp (2 \pi i c)$. Очевидно, $\operatorname{Exp}(\mathbb{R})=\mathrm{U}_{1}$. Точная последовательность

$$
0 \longrightarrow \mathbb{Z} \stackrel{j}{\longrightarrow} \mathbb{C} \stackrel{\operatorname{Exp}}{\longrightarrow} \mathbb{C}^{\times} \longrightarrow 1
$$

порождает следующую точную последовательность когомологий:

$$
0 \longrightarrow H^{1}(M, \mathbb{Z}) \stackrel{j^{*}}{\longrightarrow} H^{1}(M, \mathbb{C}) \stackrel{\operatorname{Exp}^{*}}{\longrightarrow} H^{1}\left(M, \mathbb{C}^{\times}\right) \stackrel{\delta^{*}}{\longrightarrow} H^{2}(M, \mathbb{Z}) .
$$

Хорошо известно, что $\operatorname{Ker} \delta^{*}$ - это в точности подгруппа плоских $\mathrm{GL}_{1}(\mathbb{C})$-расслоений, тривиальных как гладкие главњые расслоения. В силу предложения $1.1 \mathrm{ee}$ можно отождествить с $H_{D R}^{1}\left(M, \mathbb{C}^{\times}\right)$. Отсюда следует, что Ехр* индуцирует изоморфизм

$$
H^{1}(M, \mathbb{C}) / j^{*}\left(H^{1}(M, \mathbb{Z})\right) \rightarrow H_{D R}^{1}\left(M, \mathbb{C}^{\times}\right) .
$$

Аналогично описываются группы $H_{D R}^{1}\left(M, \mathrm{U}_{1}\right)$ и $H^{0,1}\left(M, \mathbb{C}^{\times}\right)$. При этом получаем следуюшую коммутативную диаграмму, горизонтальные стрелки которой суть изоморфизмы, индуцированные Ехр:

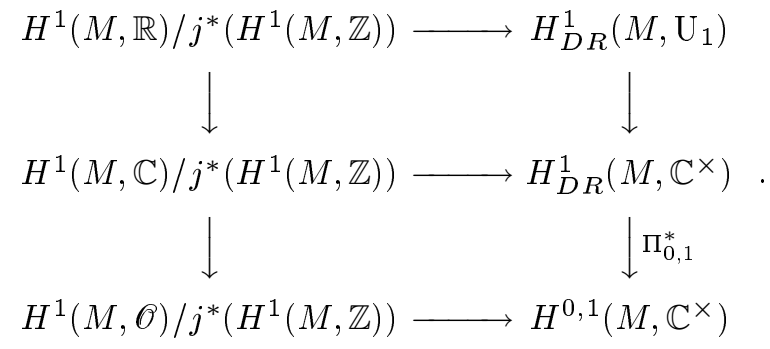

В частности, $H_{D R}^{1}\left(M, \mathbb{C}^{\times}\right)$и $H^{0,1}\left(M, \mathbb{C}^{\times}\right)$суть связные комплексные абелевы группы Ли, а $H_{D R}^{1}\left(M, \mathrm{U}_{1}\right)$ - тор. Заметим, что группа $\operatorname{Pic} M=H^{0,1}\left(M, \mathbb{C}^{\times}\right)$это по определению многообразие Пикара комплексного многообразия $M$.

По-видимому, является важной следуюшая проблема дополнения: при каких условиях отображение $\Pi_{0,1}^{*}: H_{D R}^{1}(M, G) \rightarrow H^{0,1}(M, G)$ является сюръективным? Это означает, что для любой $\alpha \in Z^{1}\left(\bar{R}_{G}\right)$ должна найтись такая форма 
$\beta \in Z^{1}\left(R_{G}\right)$, что $\Pi_{0,1} \beta=\alpha$. В п. 3.1 мы ответим на этот вопрос положительно в случае, когда $M$ - компактное кэлерово многообразие, а $G$ - борелевская подгруппа классической комплексной линейной группы (например, группа комплексных треугольных матриц $\left.T_{n}(\mathbb{C})\right)$. При этом будет использовано обобщение классической теории Ходжа. Сейчас мы рассмотрим простейший случай $G=\mathbb{C}^{\times}$ (см. п. 1.2).

ПРЕДЛОЖЕНИЕ 1.5. Пусть $M$ - компактное кәлерово многообразие. Тогда гомоморфизм $\Pi_{0,1}^{*}: H_{D R}^{1}\left(M, \mathbb{C}^{\times}\right) \rightarrow H^{0,1}\left(M, \mathbb{C}^{\times}\right)$сюргективен, а композиция гомоморфизмов

$$
H_{D R}^{1}\left(M, \mathrm{U}_{1}\right) \rightarrow H_{D R}^{1}\left(M, \mathbb{C}^{\times}\right) \stackrel{\Pi_{0,1}^{*}}{\rightarrow} H^{0,1}\left(M, \mathbb{C}^{\times}\right)
$$

из диаграммы (1.22) есть изоморфизм вещественных групп Ли.

ДокАЗАтЕльство. В силу коммутативности диаграммы (1.22) достаточно доказать аналогичные свойства для гомоморфизмов

$$
H_{D R}^{1}(M, \mathbb{R}) \rightarrow H_{D R}^{1}(M, \mathbb{C}) \stackrel{\Pi_{0,1}^{*}}{\rightarrow} H^{0,1}(M, \mathbb{C})
$$

Используя классическую теорию Ходжа (см. [9; гл. 0]), мы можем заменить в (1.23) пространства когомологий соответствуюшими пространствами гармонических форм:

$$
\mathbb{H}_{\mathbb{R}}^{1} \rightarrow \mathbb{H}_{\mathbb{C}}^{1} \stackrel{\Pi_{0,1}}{\rightarrow} \mathbb{H}^{0,1}
$$

При этом $\mathbb{H}_{\mathbb{R}}^{1}$ отождествляется с подпространством в $\mathbb{H}_{\mathbb{C}}^{1}$, состоящим из форм вида $\omega-\bar{\omega}, \omega \in \mathbb{H}^{0,1}$. Отсюда следует наше утверждение.

В качестве следствия мы получаем следующий хорошо известньй факт: группа Ріс $M$ любого компактного кэлерова многообразия $M$ есть комплексный тор.

Приведенное вьше доказательство показьвает, что любой класс когомологий из $H_{D R}^{1}\left(M, \mathrm{U}_{1}\right), H_{D R}^{1}\left(M, \mathbb{C}^{\times}\right)$или $H^{0,1}\left(M, \mathbb{C}^{\times}\right)$содержит гармоническую форму. Заметим также, что изоморфизм, обратный к $\Pi_{0,1}^{*}$, задается на уровне коциклов формулой $\omega \mapsto \omega-\bar{\omega}, \omega \in \mathbb{H}^{0,1}$.

\section{§2. Когомологии со значениями в некоторых плоских векторных расслоениях}

2.1. Пусть $\mathbf{V}$ - плоское комплексное векторное расслоение над комплексным многообразием $M$. Тогда мы имеем комплексы $\mathbf{V}$-значных форм $\left(A_{\mathbf{V}}, d\right),\left(A_{\mathbf{V}}, \partial\right)$ и $\left(A_{\mathbf{V}}, \bar{\partial}\right)$ (см. п. 1.2). Предположим, что $M$ - компактное эрмитово многообразие и что структурной группой расслоения $\mathbf{V}$ является $\mathrm{U}_{n}$. Тогда на $\mathbf{V}$ можно определить плоскую эрмитову метрику. Эта метрика позволяет определить в комплексе $A_{\mathbf{V}}$ операторы Лапласа $\Delta, \Delta_{\partial}$ и $\Delta \bar{\partial}$, заданные формулами

$$
\Delta=d d^{*}+d^{*} d, \quad \Delta_{\partial}=\partial \partial^{*}+\partial^{*} \partial, \quad \Delta \bar{\partial}=\bar{\partial}^{*}+\bar{\partial}^{*} \bar{\partial},
$$


где $d^{*}, \partial^{*}, \bar{\partial}^{*}$ - операторы, формально сопряженные с $d, \partial, \bar{\partial}$ соответственно. Обозначим через

$$
\begin{aligned}
\mathbb{H}^{r} & =(\operatorname{Ker} \Delta) \cap A_{\mathbf{V}}^{r}, \\
\mathbb{H}_{\partial}^{p, q} & =\left(\operatorname{Ker} \Delta_{\partial}\right) \cap A_{\mathbf{V}}^{p, q}, \\
\mathbb{H}_{\bar{\partial}}^{p, q} & =\left(\operatorname{Ker} \Delta_{\bar{\partial}}\right) \cap A_{\mathbf{V}}^{p, q}
\end{aligned}
$$

векторные пространства гармонических форм. Любая гармоническая форма $\omega$ является соответственно $d$-, $\partial$ - или $\bar{\partial}$-замкнутой. По теореме Ходжа соответствие $\omega \mapsto[\omega]$ определяет следующие изоморфизмы:

$$
\begin{aligned}
\mathbb{H}^{r} & \simeq H_{D R}^{r}(M, \mathbf{V}) \simeq H^{r}\left(M, \mathscr{C}_{\mathbf{V}}\right) \\
\mathbb{H}_{\partial}^{p, q} & \simeq \bar{H}^{p, q}(M, \mathbf{V}) \simeq \bar{H}^{q}\left(M, \Omega_{\mathbf{V}}^{p}\right) \\
\mathbb{H}_{\bar{\partial}}^{p, q} & \simeq H^{p, q}(M, \mathbf{V}) \simeq H^{q}\left(M, \Omega_{\mathbf{V}}^{p}\right)
\end{aligned}
$$

Мы будем использовать следующий факт, хорошо известный для обычных комплексных дифференциальных форм (см. [9; гл. 0]). В рассматриваемом случае доказательство вполне аналогично классическому (см. [1]).

ПРЕДЛОЖЕНИЕ 2.1. Предположим, что $M$ - компактное кәлерово многообразие. Тогда

$$
\Delta=2 \Delta_{\partial}=2 \Delta_{\bar{\partial}}
$$

Отсюда следует, что

$$
\mathbb{H}^{r}=\bigoplus_{p+q=r} \mathbb{H} \frac{p, q}{\bar{\partial}}, \quad \mathbb{H} \bar{p}^{\frac{p}{\partial} q}=\mathbb{H}_{\partial}^{p, q}
$$

В дальнейшем (в условиях предложения 2.1) мы будем опускать индексы $\partial$ и $\bar{\partial}$ в обозначениях для пространств гармонических форм.

СлЕДСТВИЕ 1. В условиях предложсения 2.1 векторные пространства $\mathbb{H}^{p, 0}$ $u \mathbb{H}^{0, q}$ совпадают с пространствами голоморфных $\mathbf{V}$-значных р-форм и антиголоморфных $\mathbf{V}$-значных q-форм соответственно.

СлЕДСТВИЕ 2. При тех же предположсениях имеем

$$
\begin{aligned}
& H_{D R}^{r}(M, \mathbf{V}) \simeq \bigoplus_{p+q=r} H^{p, q}(M, \mathbf{V}) \simeq \bigoplus_{p+q=r} \bar{H}^{p, q}(M, \mathbf{V}) \\
& H^{r}\left(M, \mathscr{C}_{\mathbf{V}}\right) \simeq \bigoplus_{p+q=r} H^{p}\left(M, \Omega_{\mathbf{V}}^{q}\right) \simeq \bigoplus_{p+q=r} H^{p}\left(M, \bar{\Omega}_{\mathbf{V}}^{q}\right)
\end{aligned}
$$

Рассуждая так же, как в доказательстве леммы из [9; гл. $1, \S 2]$, можно вывести из предложения 2.1 следуюшую “др-лемму”: 
СЛЕДСТВИЕ 3. Пусть V - плоское комплексное векторное расслоение со структурной группой $\mathrm{U}_{n}$ над компактным кәлеровым многообразием. Пред-

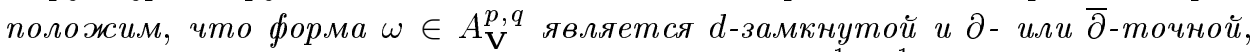
причем $p, q \geqslant 1$. Тогда существует такая $\psi \in A_{\mathbf{V}}^{p-1, q-1}$, ито $\omega=\partial \bar{\partial} \psi$.

Пусть $G \subset \mathrm{GL}_{n}(\mathbb{C})$ - некоторая линейная комплексная группа Ли, и пусть $z=\left(z_{i j}\right) \in Z^{1}(\mathfrak{U}, G)$. Рассмотрим плоское расслоение на групшы $E$, соответствующее коциклу Int $z$. Как было отмечено в п. $1.2, E$ можно рассматривать как подрасслоение плоского векторного расслоения End $\mathbf{V}(z)$ со слоем End $\mathbb{C}^{n}$, определяемого коциклом Int $z=\operatorname{Ad} z$. Применяя к последнему расслоению следствие 1 , получаем

СЛЕДСТВИЕ 4. Предположимм, что $z_{i j} \in \mathrm{U}_{n}$ u что $M$ - компактное кәлерово многообразие. Если $а \in F_{E}$ удовлетворяет условию $\Pi_{1,0} \delta_{0}(a)=0$ или $\Pi_{0,1} \delta_{0}(a)=0$, то $\delta_{0}(a)=0$, m.е. $а$ - плоское сечение.

2.2. Пусть $G$ - связная разрешимая комплексная линейная алгебраическая группа. Хорошо известно (см., например, [10; $§ 4.2])$, что $G$ допускает полупрямое разложение $G=N \rtimes S$, где $N$ - нормальная унипотентная алгебраическая группа (унипотентный радикал) групшы $G$, а $S \simeq\left(\mathbb{C}^{\times}\right)^{n}$ - алгебраический тор. Соответственно для касательной алгебры Ли $\mathfrak{g}$ группы $G$ имеем полупрямое разложение $\mathfrak{g}=\mathfrak{n} \boxplus \mathfrak{s}$, где $\mathfrak{n}$ и $\mathfrak{s}$ - идеал и подалгебра в $\mathfrak{g}$, соответствующие подгруппам $N$ и $S$. Обозначим через $p: G \rightarrow S$ естественную проекцию и через $q: S \rightarrow G$ - естественное вложение.

Пусть $K$-компактная вещественная форма тора $S$. Очевидно, $K \simeq \mathrm{U}_{1}^{n}$. Мы будем также рассматривать вешественную алгебраическую подгруппу $G_{K}=N \rtimes K$ групшы $G$.

Наиболее важным примером является подгруппа $G=T_{n}(\mathbb{C})$ в $\mathrm{GL}_{n}(\mathbb{C})$, состоящая из верхних треугольных матриц. В этом случае $N=T_{n}^{0}(\mathbb{C})$ - это нормальная подгруппа унипотентных верхних треугольных матриц, а $S=D_{n}(\mathbb{C})$ - подгруппа диагональных матриц, естественно отождествляемая с $\left(\mathbb{C}^{\times}\right)^{n}$. Алгебра Ли $\mathfrak{g}=\mathfrak{t}_{n}(\mathbb{C})$ есть подалгебра верхних треугольных матриц в $\mathfrak{g l}_{n}(\mathbb{C})$. Далее, $\mathfrak{n}-$ это идеал нильпотентных верхних треугольных матриц, а $\mathfrak{s}$ - подалгебра диагональных матриц, естественно отождествляемая с $\mathbb{C}^{n}$. Кроме того, $K$ есть подгруппа унитарных диагональных матриц, отождествляемая с $\mathrm{U}_{1}^{n}$, а соответствующая ей подалгебра Ли k $\subset \mathfrak{s}-$ это подалгебра чисто мнимых диагональных матриц. Комплекс де Рама $A_{\mathfrak{u}}$ отождествляется с комплексом s-значных форм $\gamma$, удовлетворяющих условию $\bar{\gamma}=-\gamma$. Подгруппа $G_{K}=T_{n}(\mathbb{C})_{K}$ в $T_{n}(\mathbb{C})$ состоит из матрищ с унитарной главной диагональю.

Пусть $G$ - произвольная связная разрешимая комплексная линейная алгебраическая группа, и пусть $V$ - алгебраический $G$-модуль, т.е. задано полиномиальное представление $\phi: G \rightarrow \mathrm{GL}(V)$. Пусть $\mathfrak{U}$ - открытое покрытие комплексного многообразия $M$ и $z \in Z^{1}\left(\mathfrak{U}, G_{K}\right)$. Рассмотрим соответствуюшее плоское векторное расслоение $\mathbf{V}(z)$ ранга $n$ над $M$ со структурной группой $G_{K}$. Тогда мы имеем комплекс де Рама $\left(A_{\mathbf{V}(z)}, d\right)$, а также комплексы Дольбо $\left(A_{\mathbf{V}(z)}, \bar{\partial}\right)$ и $\left(A_{\mathbf{V}(z)}, \partial\right)$.

Проекция $p: G_{K} \rightarrow K$ индуцирует отображение $p^{*}: H^{1}\left(M, G_{K}\right) \rightarrow H^{1}(M, K)$. Последнее легко описывается на уровне коцепей. А именно, заданный коцикл 
$z=\left(z_{i j}\right)$ представляется в виде $z_{i j}=u_{i j} n_{i j}$, где $u_{i j} \in K, n_{i j} \in N$. Поскольку $u_{i j}=p\left(z_{i j}\right)$, мы видим, что $u \in Z^{1}(\mathfrak{U}, K)$ и что $p^{*}$ отображает класс когомологий коцикла $z$ в класс коцикла $u$.

Предположим теперь, что $z_{i j}=g_{i}^{-1} g_{j}$ в $U_{i} \cap U_{j} \neq \varnothing$, где $g_{i}: U_{i} \rightarrow G_{K}-$ гладкие функции. Тогда $\mathbf{V}(z)$ тривиально как гладкое векторное расслоение со структурной группой $G_{K}$. Имеем $g_{i}=u_{i} n_{i}$, где $u_{i}: U_{i} \rightarrow K, n_{i}: U_{i} \rightarrow N$, откуда

$$
\begin{aligned}
u_{i j} & =p\left(g_{i}\right)^{-1} p\left(g_{j}\right)=u_{i}^{-1} u_{j}, \\
z_{i j} & =n_{i}^{-1} u_{i j} n_{j} .
\end{aligned}
$$

В частности, мы видим, что 0-коцеп $\left(n_{i}\right)$ определяет изоморфизм плоских векторных расслоений $\mathbf{V}(z)$ и $\mathbf{V}(u)$ в категории гладких векторных расслоений со структурной группой $G_{K}$. В следуюшей лемме будет предполагаться, что эта коцеп удовлетворяет условию $\Pi_{0,1} \delta_{0}\left(n_{i}\right)=0$ для всех $i$; это означает, что соответствующий изоморфизм антиголоморфен.

ЛЕмма 2.1. Пусть $z \in Z^{1}\left(\mathfrak{U}, G_{K}\right)$ - коцикл Чеха на комплексном многообразии $M$, удовлетворяющий описанному выше условию тривиальности. Предположим, что $M$ - компактное кәлерово многообразие и что $\Pi_{0,1} \delta_{0}\left(n_{i}\right)=0$ для всех $i$. Тогда справедливы следующие утверждения.

(i) Любой класс когомологий из $H^{0, q}(M, \mathbf{V}(z)), q \geqslant 0$, содержит $d$-замкнутую $\mathbf{V}(z)$-значную $(0, q)$-форму.

(ii) Любая голоморфная $\mathbf{V}(z)$-значная форма $\alpha$ является d-замкнутой. Ecли, кроме того, $\alpha$ Ә-точна, то $\alpha=0$.

(iii) Если $\alpha \in A_{\mathbf{V}(z)}^{p, 0}$ удовлетворяет условию $\partial \alpha=0$ и если $\bar{\partial} \alpha=\partial \beta$ для некоторой $\beta \in A_{\mathbf{V}(z)}^{p-1,1}$, то существует такая $\gamma \in A_{\mathbf{V}(z)}^{p-1,0}$, что $\alpha+\partial \gamma$ голоморфна.

ДокАЗАТЕЛЬСтво. Будем использовать индукцию по $n=\operatorname{dim} V$. Предположим, что $n=1$. Ясно, что $\phi\left(G_{K}\right) \subset U_{1}$, и поэтому $\mathbf{V}(z)$ - плоское линейное расслоение, определяемое коциклом $\left(\phi\left(z_{i j}\right)\right)$, где $\phi\left(z_{i j}\right) \in \mathrm{U}_{1}$. Значит, мы можем применить теорию Ходжа для плоских расслоений (см. п. 2.1). Очевидно, (i) следует из того, что любой класс когомологий Дольбо содержит гармоническую форму (см. предложение 2.1), а (ii) вытекает из следствия 1 предложения 2.1. Для доказательства утверждения (iii) заметим, что $d \partial \beta=d \bar{\partial} \alpha=0$. Применяя следствие 3 того же предложения, мы видим, что $\partial \beta=\bar{\partial} \partial \gamma$, откуда $\bar{\partial}(\alpha-\partial \gamma)=0$.

Для рассуждения по индукции нам потребуется следуюшая конструкция. По теореме Ли в пространстве $V$ сушествует базис $e_{1}, \ldots, e_{n}$, в котором $\phi$ записывается матрищей вида

$$
\phi(g)=\left(\begin{array}{cc}
\phi_{0}(g) & * \\
0 & \widetilde{\phi}(g)
\end{array}\right), \quad g \in G,
$$

где $\widetilde{\phi}: G \rightarrow T_{n-1}(\mathbb{C})$ - линейное представление, для которого $\widetilde{\phi}(g) \in D_{n-1}(\mathbb{C})$, $g \in S$, и потому $\widetilde{\phi}(g) \in \mathrm{U}_{1}^{n-1}, g \in K$. Точная последовательность $G$-модулей

$$
0 \longrightarrow V^{0} \longrightarrow V \longrightarrow \widetilde{V} \longrightarrow 0
$$


где $V^{0}=\left\langle e_{1}\right\rangle, \widetilde{V}=\left\langle e_{2}, \ldots, e_{n}\right\rangle$, порождает следующие две точные последовательности плоских векторных расслоений:

$$
\begin{aligned}
& 0 \longrightarrow \mathbf{V}^{0}(z) \stackrel{\lambda}{\longrightarrow} \mathbf{V}(z) \stackrel{\varkappa}{\longrightarrow} \tilde{\mathbf{V}}(z) \longrightarrow 0 \\
& 0 \longrightarrow \mathbf{V}^{0}(z) \stackrel{\lambda}{\longrightarrow} \mathbf{V}(u) \stackrel{\varkappa}{\longrightarrow} \tilde{\mathbf{V}}(u) \longrightarrow 0
\end{aligned}
$$

Поскольку матрицы $\phi\left(u_{i j}\right)$ диагональны, последовательность (2.3) расщепима.

Для любого $q \geqslant 0$ из (2.2) получается следующая точная последовательность пучков антиголоморфных $q$-форм:

$$
0 \longrightarrow \bar{\Omega}_{\mathbf{V}^{0}(z)}^{q} \stackrel{\lambda}{\longrightarrow} \bar{\Omega}_{\mathbf{V}(z)}^{q} \stackrel{\varkappa}{\longrightarrow} \bar{\Omega}_{\tilde{\mathbf{V}}(z)}^{q} \longrightarrow 0
$$

Отображения $n_{i}$ (см. (2.1)) определяют антиголоморфньй изоморфизм последовательности (2.2) на расщепимую точную последовательность (2.3). Отсюда следует, что точная последовательность пучков (2.4) расщепима. Значит, для любого $p \geqslant 0$ мы получаем следующую расщепимую точную последовательность групп когомологий:

$$
0 \longrightarrow H^{p}\left(M, \bar{\Omega}_{\mathbf{V}^{0}(z)}^{q}\right) \stackrel{\lambda^{*}}{\longrightarrow} H^{p}\left(M, \bar{\Omega}_{\mathbf{V}(z)}^{q}\right) \stackrel{\varkappa^{*}}{\longrightarrow} H^{p}\left(M, \bar{\Omega}_{\widetilde{\mathbf{V}}(z)}^{q}\right) \longrightarrow 0 .
$$

Используя изоморфизм (1.12), можно интерпретировать (2.5) как следующую расщепимую точную последовательность:

$$
0 \longrightarrow \bar{H}^{p, q}\left(M, \mathbf{V}^{0}(z)\right) \stackrel{\lambda^{*}}{\longrightarrow} \bar{H}^{p, q}(M, \mathbf{V}(z)) \stackrel{\varkappa^{*}}{\longrightarrow} \bar{H}^{p, q}(M, \tilde{\mathbf{V}}(z)) \longrightarrow 0
$$

Заметим также, что (2.2) порождает следующие расщепимые точные последовательности:

$$
0 \longrightarrow A_{\mathbf{V}^{0}(z)}^{p, q} \stackrel{\lambda}{\longrightarrow} A_{\mathbf{V}(z)}^{p, q} \stackrel{\varkappa}{\longrightarrow} A_{\widetilde{\mathbf{V}}(z)}^{p, q} \longrightarrow 0 .
$$

Перейдем теперь к индуктивному рассуждению. Предположим, что утверждения (i), (ii) и (iii) верны для векторных расслоений ранга $n-1$.

(i) Мы будем считать, что $q \geqslant 1$, поскольку в случае $q=0$ (i) совпадает с (ii). Пусть $\alpha$ - такая $\mathbf{V}(z)$-значная $(0, q)$-форма, что $\bar{\partial} \alpha=0$, причем $\operatorname{dim} V=n$. Рассмотрим точную последовательность (2.7) для $p=0$. По предположению индукции $\varkappa(\alpha)=\beta+\bar{\partial} \varphi$, где $d \beta=0$ и $\varphi \in A_{\widetilde{\mathbf{v}}(z)}^{0, q-1}$. Тогда $\varphi=\varkappa(\psi)$ для некоторой $\psi \in A_{\mathbf{V}(z)}^{0, q-1}$. Значит, $\beta=\varkappa(\alpha-\bar{\partial} \psi)$. Отсюда $\varkappa(\partial(\alpha-\bar{\partial} \psi))=0$ и $\partial(\alpha-\bar{\partial} \psi)=\lambda\left(\alpha^{\prime}\right)$, где $\alpha^{\prime}$-некоторая $d$-замкнутая $\mathbf{V}^{0}(z)$-значная $(1, q)$-форма. Используя (2.6), заключаем, что $\alpha^{\prime}$ является $\partial$-точной. Применяя следствие 3 предложения 2.1 , видим, что $\alpha^{\prime}=\partial \bar{\partial} \omega$ для некоторой $\omega \in A_{\mathbf{V}^{0}(z)}^{0, q-1}$. Отсюда следует, что $\partial(\alpha-\bar{\partial}(\psi+\lambda(\omega)))=0$. Таким образом, $\alpha-\bar{\partial}(\psi+\lambda(\omega))$ есть искомая $d$-замкнутая форма.

(ii) Пусть $\alpha$-голоморфная $\mathbf{V}(z)$-значная $p$-форма, причем $\operatorname{dim} V=n$. По предположению индукции $d \varkappa(\alpha)=0$, откуда $\varkappa(d \alpha)=\varkappa(\partial \alpha)=0$. Отсюда следует, что $\partial \alpha=\lambda(\beta)$, где $\beta$ - некоторая $\mathbf{V}^{0}(z)$-значная голоморфная $(p+1)$-форма. 
Из (2.6) заключаем, что $\beta$ является $\partial$-точной. Используя следствие 1 предложения 2.1 , примененное к $\mathbf{V}^{0}(z)$-значным формам, видим, что $\beta$ гармонична и потому $\beta=0$. Значит, $\partial \alpha=d \alpha=0$.

Предположим, что $\alpha=\partial \gamma$. Тогда $\varkappa(\alpha)=\partial \varkappa(\gamma)=0$ по предположению индукции. Значит, $\alpha=\lambda(\beta)$, где $\beta$ - некоторая $\mathbf{V}^{0}(z)$-значная голоморфная $p$-форма. Тогда из (2.6) следует, что $\beta$ является $\partial$-точной в $A_{\mathbf{V}^{0}(z)}$ и потому $\beta=0$.

(iii) Предположим, что $\partial \alpha=0$ и что $\bar{\partial} \alpha=\partial \beta$. Ясно, что $\varkappa(\alpha)$ удовлетворяет аналогичным условиям. По предположению индукции $\bar{\partial}\left(\varkappa(\alpha)+\partial \gamma_{1}\right)=0$ для некоторой $\gamma_{1} \in A_{\widetilde{\mathbf{V}}(z)}^{p-1,0}$. Используя $(2.7)$, мы можем выбрать $\gamma_{2} \in A_{\mathbf{V}(z)}^{p-1,0}$ таким образом, чтобы $\varkappa\left(\gamma_{2}\right)=\gamma_{1}$. Тогда $\varkappa\left(\bar{\partial}\left(\alpha+\partial \gamma_{2}\right)\right)=0$, откуда $\bar{\partial}\left(\alpha+\partial \gamma_{2}\right)=$ $\lambda(\varphi)$ для некоторой $\mathbf{V}^{0}(z)$-значной $(p, 1)$-формы $\varphi$. Очевидно, $d \varphi=0$ и из $(2.6)$ следует, что $\varphi$ является $\partial$-точной. В силу следствия 3 предложения $2.1 \varphi=\bar{\partial} \partial \gamma_{3}$. Следовательно, $\bar{\partial}\left(\alpha+\partial\left(\gamma_{2}-\gamma_{3}\right)\right)=0$.

СлЕДСТВИЕ. Пусть $E$ - плоское расслоение на группи со слоем $G$, определяемое коциклом Int $z$. Если сечение $a \in F_{E}$ голоморфно, то а плоско.

ДокАЗАТЕЛЬСтво. Рассуждение аналогично доказательству следствия 4 предложения 2.1. А именно, фиксируем точное голоморфное представление $\phi$ группы $G$ и рассмотрим соответствующее плоское векторное расслоение $\mathbf{V}(z)$. Тогда $E$ есть подрасслоение плоского векторного расслоения $\mathbf{E n d} \mathbf{V}(z)$, определяемого коциклом Int $\phi(z)$. Затем применяем утверждение (ii) к объемлющему плоскому векторному расслоению.

\section{§ 3. Когомологии со значениями в некоторых разрешимых алгебраических группах}

3.1. Пусть $M$ - компактное кэлерово многообразие. Как следует из классической теории Ходжа, любой класс когомологий из $H^{0,1}(M, \mathbb{C})$ содержит гармоническую (и следовательно, $d$-замкнутую) $(0,1)$-форму. Аналогичный факт имеет место для когомологий Дольбо со значениями в комплексном алгебраическом торе (см. предложение 1.5$)$. В частности, для групп $\mathbb{C}^{n}$ и $\left(\mathbb{C}^{\times}\right)^{n}$ проблема дополнения, сформулированная в п. 1.3, допускает положительное решение. В этом пункте мы рассмотрим класс разрешимых комплексных алгебраических групп, обладающий аналогичным свойством.

Пусть $G$ - связная разрешимая комплексная линейная алгебраическая группа. Мы будем использовать полупрямое разложение $G=N \rtimes S$, описанное в п. 2.2, сохраняя обозначения, введенные в этом пункте. Общие свойства когомологий де Рама и Дольбо со значениями в полупрямом произведении были установлены в [8; теоремы 2.2 и 2.4]. В частности, имеется следуюшая коммутативная диаграмма, строки которой точны:

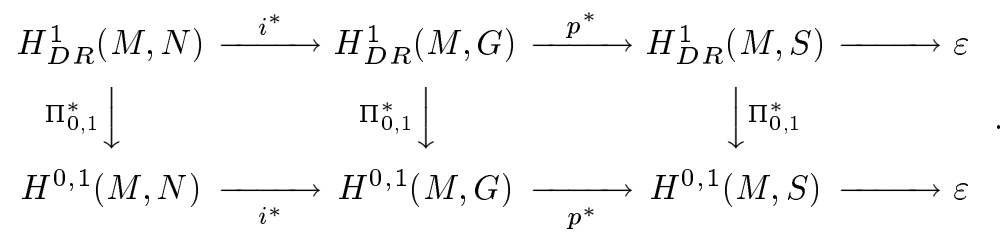


Отображения $i^{*}$ индуцированы вложением $i: N \rightarrow G$. Мы также имеем инъективные отображения $q^{*}: H_{D R}^{1}(M, S) \rightarrow H_{D R}^{1}(M, G)$ и $q^{*}: H^{0,1}(M, S) \rightarrow H^{0,1}(M, G)$ такие, что $p^{*} \circ q^{*}=\mathrm{id}$. Будем отождествлять $H_{D R}^{1}(M, S)$ и $H^{0,1}(M, S)$ с их образами в $H_{D R}^{1}(M, G)$ и $H^{0,1}(M, G)$ соответственно. Поскольку $K$ есть прямой сомножитель группы $S$, имеется также вложение $H_{D R}^{1}(M, K) \subset H_{D R}^{1}(M, S)$.

В силу предложения $1.5 \Pi_{0,1}^{*}$ индуцирует изоморфизм групп $H_{D R}^{1}(M, K) \rightarrow$ $H^{0,1}(M, S)$; он соответствует изоморфизму пространств $\mathfrak{k}$-значных гармонических 1-фором и s-значных гармонических $(0,1)$-форм $\mathbb{H}_{\mathfrak{k}}^{1} \rightarrow \mathbb{H}_{\mathfrak{s}}^{0,1}$, заданному формулой $\chi-\bar{\chi} \mapsto \chi$

Построим теперь некоторые подкрученные комплексы де Рама и Дольбо (см. п. 1.2). Фиксируем гармоническую форму $\gamma=-\bar{\chi}+\chi \in \mathbb{H}_{\mathfrak{k}}^{0,1}$, где $\chi \in \mathbb{H}_{\mathfrak{s}}^{0,1}$. Выберем открытое покрытие $\mathfrak{U}=\left(U_{i}\right)$ многообразия $M$ такое, что $\gamma=\delta_{0}\left(u_{i}\right)$ в каждом $U_{i}$ для некоторых гладких функций $u_{i}: U_{i} \rightarrow K$, и построим коцикл Чеха $z=\left(z_{i j}\right)$, где $z_{i j}=u_{i}^{-1} u_{j}$ в любом $U_{i} \cap U_{j} \neq \varnothing$. Фиксируем также функции $u_{i}$ и тем самьм коцикл $z$. Обозначим через $E$ плоское расслоение на группы, полученное из $M \times G$ путем подкручивания при помощи коцикла Int $z$, и через $\mathfrak{e}-$ coответствуюшее расслоение на алгебры Ли. Так как $z$ принимает значения в $K$, то мы получаем также нормальное подрасслоение на группы $E_{N} \subset E$ со слоем $N$, соответствующее коциклу Int $z \mid N$, причем $E$ будет полупрямым произведением расслоения $E_{N}$ и тривиального расслоения $M \times S$. Соответственно получаем полупрямое разложение $A_{\mathfrak{e}}=A_{\mathfrak{e}_{\mathfrak{n}}} \boxplus A_{\mathfrak{s}}$. Расслоения $E$ и $E_{N}$ мы будем называть унитарными плоскими расслоениями на группь со слоями $G$ и $N$ соответственно, связанными с формой $\gamma$.

Рассмотрим подкрученные комплексы де Рама и Дольбо $R_{E}$ и $\bar{R}_{E}$. В силу предложения 1.3 отображение $r_{\gamma}: A_{\mathfrak{e}}^{1} \rightarrow A_{\mathfrak{g}}^{1}$, определенное в п. 1.2 , индуцирует биекцию $r_{\gamma}^{*}: H_{D R}^{1}(M, E) \rightarrow H_{D R}^{1}(M, G)$ такую, что $r_{\gamma}^{*}(\varepsilon)=\zeta$ есть класс когомологий формы $\gamma$. Аналогично (см. предложение 1.4 ) имеем отображение $\bar{r}_{\chi}: A_{\mathfrak{e}}^{0,1} \rightarrow A_{\mathfrak{g}}^{0,1}$, индуцируюшее биекцию $\bar{r}_{\chi}^{*}: H^{0,1}(M, E) \rightarrow H^{0,1}(M, G)$ такую, что $\bar{r}_{\chi}^{*}(\varepsilon)=\xi$ есть класс когомологий формы $\chi$. При этом имеет место следующая коммутативная диаграмма с точными строками (см. [8]):

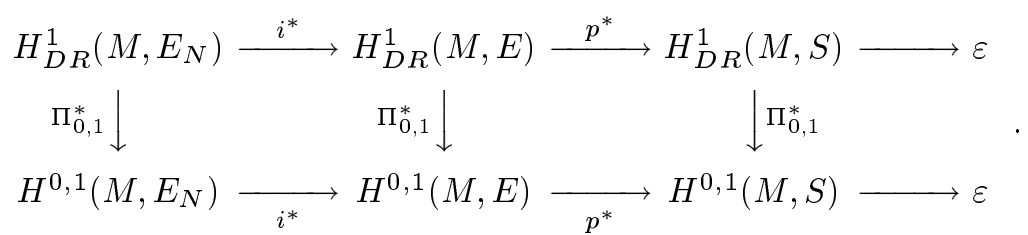

Здесь $H_{D R}^{1}(M, S)$ и $H^{0,1}(M, S)$ естественно вложены в $H_{D R}^{1}(M, E)$ и $H^{0,1}(M, E)$ соответственно. Из (1.21) видно, что правый квадрат этой диаграммы получается из правого квадрата диаграммы (3.1) при помощи биекций $r_{\gamma}^{-1}$ и $\bar{r}_{\chi}^{-1}$.

Будем говорить, что группа $G$ обладает свойством Ходжа, если для всякого унитарного плоского расслоения на группы $E$ со слоем $G$ над компактным кэлеровьп многообразием $M$ выполняется следующее условие: для любого $\sigma \in \operatorname{Im} i^{*}=$ $\operatorname{Ker} p^{*} \subset H^{0,1}(M, E)$ сушествует форма $\eta \in \sigma$ такая, что

$$
\eta \in A_{\mathfrak{e}_{\mathfrak{n}}}^{0,1}, \quad \delta_{1}(\eta)=0 .
$$


ПримеР 3.1. Как мы видели выше, алгебраический тор $G$ обладает свойством Ходжа. То же верно при более общем предположении, что унипотентный идеал $N$ группы $G$ абелев. Действительно, в этом случае любое унитарное плоское расслоение $E_{N}$ со слоем $N$ есть плоское векторное расслоение с унитарной структурной группой. В силу предложения 2.1 любой класс из $H^{0,1}\left(M, E_{N}\right)$ содержит $d$-замкнутую форму. Отсюда легко следует наше утверждение.

Переформулируем теперь свойство Ходжа на языке обычных комплексов де Рама и Дольбо.

ПРЕДЛОЖЕНИЕ 3.1. Группа $G$ обладает свойством Ходжа тогда и только тогда, когда для любого компактного кәлерова многообразия $M$, любого фиксированного класса $\sigma \in H^{0,1}(M, G)$ и любой фиксированной гармонической формы $\chi \in \mathbb{H}_{\mathfrak{s}}^{0,1}$, представляющей класс $p^{*}(\sigma)$, существует форма $\beta \in Z^{1}\left(R_{G}\right)$ такая, что $\Pi_{0,1} \beta \in \sigma$, причем

$$
\beta_{\mathfrak{s}}=\chi-\bar{\chi}, \quad \beta_{\mathfrak{n}} \in A_{\mathfrak{n}}^{0,1} .
$$

ДокАЗАТЕЛЬСтво. Пусть заданы класс $\sigma \in H^{0,1}(M, G)$ и гармоническая форма $\chi \in \mathbb{H}_{\mathfrak{s}}^{0,1}$. Положим $\gamma=-\bar{\chi}+\chi$ и рассмотрим класс $\widetilde{\sigma}=\left(\bar{r}_{\chi}^{*}\right)^{-1}(\sigma) \in$ $H^{0,1}(M, E)$, где $E$ - унитарное плоское расслоение на группы, соответствующее форме $\gamma$. Очевидно, $p^{*}(\sigma)=[\chi]$ тогда и только тогда, когда $p^{*}(\widetilde{\sigma})=\varepsilon$. Предположим, что $\eta \in \widetilde{\sigma}$ удовлетворяет (3.3), и рассмотрим форму $\beta=r_{\gamma}(\eta) \in Z^{1}\left(R_{G}\right)$. Очевидно, $\beta=\operatorname{Ad} u_{i}\left(\eta_{i}\right)+\gamma$ удовлетворяет $(3.4)$ и $\left[\Pi_{0,1} \beta\right]=\bar{r}_{\chi}^{*}[\widetilde{\sigma}]=[\sigma]$. Обратно, если $\beta \in Z^{1}\left(R_{G}\right)$ удовлетворяет (3.4) и $\Pi_{0,1} \beta \in \sigma$, то $\eta=r_{\gamma}^{-1}(\beta)$ удовлетворяет (3.3) и лежит в классе $\widetilde{\sigma}$.

СлЕДСТВИЕ. Если группа $G$ обладает свойством Ходжса, то проблема дополнения для $G$ имеет поломительное решение на любом компактном кәлеровом многообразии.

Фиксируем теперь форму $\beta \in Z^{1}\left(R_{G}\right)$, удовлетворяющую (3.4). Эту форму также можно использовать для подкручивания комплекса $R_{G}$. Запишем $\beta=\delta_{0}\left(b_{i}\right)$ для некоторых гладких функций $b_{i}: U_{i} \rightarrow G$ в любом $U_{i}$. Эти функции можно выбрать так, чтобы $b_{i}=u_{i} n_{i}$ для некоторых гладких функций $n_{i}: U_{i} \rightarrow N$. Действительно, для формы $\eta=r_{\gamma}^{-1}(\beta)$ имеем $\eta_{i}=\rho\left(u_{i}\right)^{-1}(\beta)=\rho\left(u_{i}\right)^{-1}\left(\delta_{0}\left(b_{i}\right)\right)=$ $\delta_{0}\left(u_{i}^{-1} b_{i}\right)$. С другой стороны, $\eta_{i}=\delta_{0}\left(n_{i}\right)$ для некоторых гладких $n_{i}: U_{i} \rightarrow N$. Значит, $n_{i}=u_{i}^{-1} b_{i} g_{i}$, где $g_{i}: U_{i} \rightarrow G$ удовлетворяют условию $\delta_{0}\left(g_{i}\right)=0$. Тогда $b_{i} g_{i}=u_{i} n_{i}$ и $\delta_{0}\left(b_{i} g_{i}\right)=0$. Заменяя $b_{i}$ на $b_{i} g_{i}$, получаем требуемьй результат. Построим теперь коцикл Чеха $w=\left(w_{i j}\right) \in Z^{1}(\mathfrak{U}, G)$, где

$$
w_{i j}=b_{i}^{-1} b_{j}=n_{i}^{-1} z_{i j} n_{i} .
$$

Рассмотрим плоское расслоение на группы $E^{\prime}$ и плоское расслоение на алгебры Ли $\mathfrak{e}^{\prime}$ со слоями $G$ и $\mathfrak{g}$, определяемое коциклами $\left(\operatorname{Int} w_{i j}\right)$ и $\left(\operatorname{Ad} w_{i j}\right)$ соответственно. Очевидно, $\beta=\delta_{0}\left(u_{i} n_{i}\right)=\gamma+\operatorname{Ad} u_{i}\left(\delta_{0}\left(n_{i}\right)\right)$, откуда

$$
\eta_{i}=\operatorname{Ad} u_{i}^{-1}(\beta-\gamma)=\delta_{0}\left(n_{i}\right)
$$

и $\Pi_{1,0} \delta_{0}\left(n_{i}\right)=0$. Значит, $\mathfrak{e}^{\prime}$ удовлетворяет условиям леммы 2.1 . 
Мы будем также использовать отображение $r_{\beta}: A_{\mathfrak{e}^{\prime}}^{1} \rightarrow A_{\mathfrak{g}}^{1}$. Укажем явное выражение для отображения $r_{\beta}^{-1} \circ r_{\gamma}: \psi \mapsto \psi^{\prime}$ пространства $A_{\mathfrak{e}}^{1}$ на $A_{\mathfrak{e}^{\prime}}^{1}$. Очевидно, $\rho\left(u_{i}\right)\left(\psi_{i}\right)=r_{\gamma}(\psi)=r_{\beta}\left(\psi^{\prime}\right)=\rho\left(u_{i} n_{i}\right)\left(\psi_{i}^{\prime}\right)$, откуда

$$
\begin{aligned}
\psi_{i} & =\rho\left(n_{i}\right)\left(\psi_{i}^{\prime}\right), \\
\psi_{i}^{\prime} & =\operatorname{Ad} n_{i}^{-1}\left(\psi_{i}-\eta_{i}\right) .
\end{aligned}
$$

Теперь мы хотим доказать, что некоторые разрешимые алгебраические группы $G$ обладают свойством Ходжа. Ключевую роль будет играть следующий результат.

Лемма 3.1. Пусть $G=B \rtimes A$, әде $B$ - абелева унипотентная нормальная алгебрачческая подгруппа и $A$ - связная разрешимая алгебраическая подгруппа, обладающая свойством Ходжса. Тогда $G$ обладает свойством Ходжса.

ДоказАТЕльство. Имеем полупрямое разложение $A=N_{0} \rtimes S$, где $N_{0}$ - унипотентный радикал групшы $A$ и $S$ - алгебраический тор. Тогда $G=N \rtimes S$, где $N=B \rtimes N_{0}$. Ясно, что $N-$ унипотентная алгебраическая подгруппа, а потому и унипотентный радикал групшы $G$.

Пусть теперь $M$ - компактное кэлерово многообразие. Фиксируем гармоническую форму $\chi \in A_{\mathfrak{s}}^{0,1}$, обозначим $\gamma=-\bar{\chi}+\chi$ и построим соответствующий коцикл Чеха $z$ и унитарное плоское расслоение на группы $E$ над $M$. Выберем класс $\sigma \in \operatorname{Ker} p^{*} \subset H^{0,1}(M, E)$. Тогда $\sigma=[\alpha]$ для некоторой $\alpha \in Z^{1}\left(\bar{R}_{E_{N}}\right)$. Так как $A$ и $B$ инвариантны относительно Int $z_{i j}$, то $E_{N}=E_{B} \rtimes E_{N_{0}}$, где $E_{B}$ и $E_{N_{0}}$ - подрасслоения, отвечающие подгруппам $B$ и $N_{0}$ в $N$. Соответственно имеем $A_{\mathfrak{e}_{\mathfrak{n}}}^{0,1}=A_{\mathfrak{e}_{\mathfrak{b}}}^{0,1} \boxplus A_{\mathfrak{e}_{\mathfrak{n}_{0}}}^{0,1}$, где $\mathfrak{e}_{\mathfrak{b}}$ и $\mathfrak{e}_{\mathfrak{n}_{0}}-$ соответствующие подрасслоения в $\mathfrak{e}_{\mathfrak{n}}$. Поэтому $\alpha=\alpha_{\mathfrak{b}}+\alpha_{\mathfrak{e}_{\mathfrak{n}_{0}}}$, где $\alpha_{\mathfrak{b}} \in A_{\mathfrak{e}_{\mathfrak{b}}}^{0,1}$, a $\alpha_{\mathfrak{e}_{\mathfrak{n}_{0}}} \in Z^{1}\left(\bar{R}_{E_{N_{0}}}\right)$. Так как $A$ обладает свойством Ходжа, то форма $\alpha_{\mathfrak{e}_{\mathfrak{n}_{0}}}$ когомологична в $\bar{R}_{E_{A}}$ некоторой форме $\eta_{\mathfrak{a}} \in Z^{1}\left(R_{E_{N_{0}}}\right)$. Заменяя $\alpha$ когомологичным коциклом, мы можем считать, что $\alpha_{\mathfrak{e}_{\mathfrak{n}_{0}}}=\eta_{\mathfrak{a}}$.

Рассмотрим теперь форму $\beta_{\mathfrak{a}}=r_{\gamma}\left(\eta_{\mathfrak{a}}\right) \in Z^{1}\left(R_{A}\right)$. Очевидно, $\eta_{\mathfrak{a}} \in R_{E}^{1}$ удовлетворяет (3.3). Поэтому мы можем применить изложенную выше конструкцию плоского расслоения на группы $E^{\prime}$ со слоем $G$, используя форму $\beta_{\mathfrak{a}}$ в качестве $\beta$. В частности, можно записать $\beta_{\mathfrak{a}}=\delta_{0}\left(a_{i}\right)$ в любом $U_{i}$, где $a_{i}=u_{i} n_{i}$ для некоторых гладких функций $n_{i}: U_{i} \rightarrow N_{0}$, удовлетворяющих условиям $\Pi_{1,0} \delta_{0}\left(n_{i}\right)=0$. Тогда $E^{\prime}$ определяется коциклом $w \in Z^{1}\left(\mathfrak{U}, N_{0} \rtimes K\right)$, заданным формулой $w_{i j}=a_{i}^{-1} a_{j}$. Поскольку $w_{i j} \in A$, имеем $E^{\prime}=E_{B}^{\prime} \rtimes E_{A}^{\prime}$, где $E_{B}^{\prime}$ и $E_{A}^{\prime}$ - подрасслоения, отвечающие подгруппам $B$ и $A$ групшы $G$. Используя $(3.5)$, получаем

$$
\alpha^{\prime}=\operatorname{Ad} n_{i}^{-1}\left(\alpha_{i}-\eta_{i}\right)=\operatorname{Ad} n_{i}^{-1}\left(\left(\alpha_{\mathfrak{b}}\right)_{i}\right)
$$

откуда

$$
\bar{r}_{\Pi_{0,1} \beta_{\mathfrak{a}}}^{-1}\left(\bar{r}_{\chi}(\alpha)\right)=\Pi_{0,1} \alpha^{\prime}=\alpha^{\prime}=\operatorname{Ad} n_{i}^{-1}\left(\left(\alpha_{\mathfrak{b}}\right)_{i}\right)
$$

Итак, $\alpha^{\prime} \in Z^{1}\left(\bar{R}_{E_{B}^{\prime}}\right)$. Поскольку $B$ абелева, $E_{B}^{\prime}$ есть плоское векторное расслоение, определенное коциклом Int $w_{i j} \mid B$ и потому удовлетворяющее условиям леммы 2.1. В силу утверждения (i) этой леммы $\alpha^{\prime}$ когомологична в $\bar{R}_{E_{B}^{\prime}}$ некоторой 
$(0,1)$-форме $\alpha_{1}^{\prime}$ такой, что $d \alpha_{1}^{\prime}=\delta\left(\alpha_{1}^{\prime}\right)=0$. Согласно $(3.5)$ соответствуюшая форма $\alpha_{1} \in Z^{1}\left(R_{E}\right)$ имеет вид

$$
\left(\alpha_{1}\right)_{i}=\rho\left(n_{i}\right)\left(\left(\alpha_{1}^{\prime}\right)_{i}\right)=\operatorname{Ad} n_{i}\left(\left(\alpha_{1}^{\prime}\right)_{i}\right)+\delta_{0}\left(n_{i}\right)
$$

Поскольку $\Pi_{1,0} \delta_{0}\left(n_{i}\right)=0$, эта форма удовлетворяет (3.3). Ясно, что $\alpha_{1} \in \sigma$ и лемма 3.1 доказана.

Мы будем также использовать следуюшее замечание.

ЗАмЕчАнИЕ 3.1. Из определения свойства Ходжа непосредственно следует, что некоторые несложные модификации разрешимой алгебраической групшы $G$ сохраняют это свойство.

1) Если $G=N \rtimes S$ обладает свойством Ходжа, то то же верно для группы $\widetilde{G}=N \rtimes \widetilde{S}$, где $\widetilde{S}-$ любой подтор в $S$.

2) Пусть $Z \subset S$ - центральная алгебраическая подгруппа в $G=N \rtimes S$. Группа $G$ обладает свойством Ходжа тогда и только тогда, когда это верно для $G / Z$.

3) Если $G_{1}$ и $G_{2}$ обладают свойством Ходжа, то тем же свойством обладает $G_{1} \times G_{2}$.

Теорема 3.1. Пусть $G$ - борелевская подгруппа редуктивной комплексной алгебраической группы $\widehat{G}$, все неабелевы простые факторы которой имеют mun $A_{n}, B_{n}, C_{n}, D_{n}, E_{6}$ или $E_{7}$. Тогда $G$ обладает свойством Ходжа.

ДокАЗАТЕЛЬСТво. Воспользуемся индукцией по рангу (т.е. размерности максимального алгебраического подтора) $n$ групшы $\widehat{G}$. Допустим, что наше утверждение доказано для групा $\widehat{G}$ рангов $<n$, удовлетворяющих условиям теоремы, и пусть $G$ - борелевская подгруппа редуктивной группы $\widehat{G}$ ранга $n$, все неабелевы простые факторы которой имеют тип $A_{n}, B_{n}, C_{n}, D_{n}, E_{6}$ или $E_{7}$. Используя предположение индукции и замечание 3.1 , видим, что достаточно рассмотреть случай, когда $\widehat{G}$ - простая группа одного из указанных типов. Хорошо известно (см. [11]), что любая из этих групा $\widehat{G}$ содержит такую параболическую подгруппу $P$, что $\widehat{G} / P$ - эрмитово симметрическое пространство, что равносильно абелевости унипотентного радикала $B$ группы $P$. При этом $P=B \rtimes Q$, где $Q=H \mathbb{C}^{\times}$ - локально прямое произведение, $H$ - полупростая подгруппа ранга $n-1$. Мы можем считать, что $G \subset P$, откуда $G=B \rtimes\left(G_{1} \mathbb{C}^{\times}\right)$, где $G_{1}$ - борелевская подгруппа группы $H$. Схема Дьнкина группы $H$ получается из схемы Дынкина группы $\widehat{G}$ при отбрасьвании одной из вершин, так что $H$ содержит только простые факторы классических типов или типа $E_{6}$ (для $\widehat{G}=E_{7}$, см. [11]). Применяя предположение индукции, видим, что $G_{1}$ обладает свойством Ходжа. Теперь теорема следует из замечания 3.1 и леммы 3.1 .

СледСтвиЕ. Группа $T_{n}(\mathbb{C})$ обладает свойством Ходжса.

ЗАмечание 3.2 . Остающиеся простые группы $G_{2}, F_{4}$ и $E_{8}$ не содержат параболических подгрупп с абелевым унипотентным радикалом. Нам неизвестно, обладают ли свойством Ходжа их борелевские подгруппы. 
3.2. Теперь будем предполагать, что $M$ - компактное кэлерово многообразие, а $G$ - связная разрешимая комплексная алгебраическая группа, обладающая свойством Ходжа. Наша цель состоит в изучении множества $H_{D R}^{1}(M, G)$.

Мы сохраняем обозначения, введенные в п. 3.1. Фиксируем класс $\xi \in H^{0,1}(M, S)$, гармоническую форму $\chi \in A_{\mathfrak{s}}^{0,1}$, представляющую этот класс, и коцикл Чеха $z=\left(z_{i j}\right)$ со значениями в $K$, соответствующий форме $\gamma=-\bar{\chi}+\chi$. При изучении множества $H_{D R}^{1}(M, G)$ удобно заменить $R_{G}$ подкрученньп комплексом $R_{E}$, где $E$ - соответствующее плоское унитарное расслоение на группы.

Согласно определению свойства Ходжа любой класс $\sigma \in H^{0,1}(M, E)$, для которого $p^{*} \sigma=\varepsilon$, содержит форму $\eta$, удовлетворяющую (3.3).

ПРЕДЛОЖЕНИЕ 3.2. Форма $\eta$ определена в своем классе когомологий комплекса $R_{E}$ однозначно с точностью до преобразования вида $\mathrm{Ad} a$, де а - плоское сечение расслоения $E$.

Форма $\eta$ гармонична и потому $d \eta=[\eta, \eta]=0$.

ДокАЗАтЕльство. Пусть $\eta_{1} \in Z^{1}\left(R_{E_{N}}\right)$ - другая $(0,1)$-форма такая, что $\eta_{1}=$ $\rho(a)(\eta)=\operatorname{Ad} a(\eta)+\delta_{0}(a)$, где $a \in F_{E}$. Тогда, очевидно, $\Pi_{1,0} \delta_{0}(a)=0$. В силу следствия 4 предложения $2.1 \delta_{0}(a)=0$.

Из условия $\delta_{1}(\eta)=0$ следует $\partial \eta=0$. Значит, форма $\eta$ антиголоморфна и потому гармонична в силу следствия 1 предложения 2.1. Отсюда вытекает, что $d \eta=[\eta, \eta]=0$.

Теперь мы фиксируем класс когомологий $\sigma \in H^{0,1}(M, E)$ такой, что $p^{*} \sigma=$ $\varepsilon$, и форму $\eta \in \sigma$, удовлетворяюшую (3.3). Мы хотим описать подмножество $\left(\Pi_{0,1}^{*}\right)^{-1}(\sigma) \subset H_{D R}^{1}(M, E)$. Оно непусто, так как содержит класс когомологий формы $\eta$. Форму $\omega \in Z^{1}\left(R_{E}\right)$ назовем канонической, если $\Pi_{0,1} \omega \in Z^{1}\left(E_{N}\right)$. Например, $\eta$ является канонической формой.

ПРЕДЛОЖЕНИЕ 3.3. Любой Класс когомологий $\tau \in H_{D R}^{1}(M, E)$ такой, что $\Pi_{0,1}^{*}(\tau)=\sigma$, содержит коцикл $\omega$, который удовлетворяет условию $\Pi_{0,1} \omega=\eta$ и потому является канонической формой.

ДокАЗАТЕльство. В силу свойства Ходжа для любого $\alpha \in \tau$ сушествует такой $a \in F_{E}$, что

$$
\eta=\bar{\rho}(a)\left(\Pi_{0,1} \alpha\right)=\Pi_{0,1} \rho(a)(\alpha)=\Pi_{0,1} \omega
$$

где $\omega=\rho(a)(\alpha) \in \tau$.

Рассмотрим форму $\beta=r_{\gamma}(\eta)$. Для получения дальнейшей информации о канонических формах мы будем использовать подкрученный комплекс $R_{E^{\prime}}$, введенньй в п. 3.1, и соответствие $r_{\beta}^{-1} \circ r_{\gamma}: \psi \mapsto \psi^{\prime}$, описываемое формулами (3.5).

ПРЕДЛОЖЕНИЕ 3.4. Пусть $\omega-$ каноническая форма такая, что $\Pi_{0,1} \omega=\eta$. Обозначим $\alpha=\Pi_{1,0} \omega$. Тогда

(i) $\omega_{i}^{\prime}=\operatorname{Ad} n_{i}^{-1}\left(\alpha_{i}\right)$,

(ii) $\omega^{\prime}$ гармонична и удовлетворяет уравнениям $d \omega^{\prime}=\left[\omega^{\prime}, \omega^{\prime}\right]=0$,

(iii) $d \alpha=\bar{\partial} \alpha=[\eta, \alpha], \partial \alpha=0$. 
ДокАЗАТЕЛЬство. Соотношение (i) легко следует из (3.5). Так как $\omega^{\prime}-$ форма типа $(1,0)$, из равенства $\delta_{1}\left(\omega^{\prime}\right)$ следует $\bar{\partial} \omega^{\prime}=0$. Значит, $\omega^{\prime}$ голоморфна и потому гармонична в силу следствия 1 предложения 2.1. Отсюда вытекает (ii). Наконец, (iii) следует из (ii) и из последнего утверждения предложения 3.2 .

ПРЕДЛОЖЕНИЕ 3.5. Если $\omega$ и $\omega_{1}$ - две канонические формьи, принадлежащие одному и тому же классу $\tau$, то $\omega_{1}=\operatorname{Ad} f(\omega)$ для некоторого $f \in H^{0}\left(M, \mathscr{C}_{E}\right)$.

ДокАЗАТЕЛЬСТВо. В силу предложения 3.3 можно считать, что $\Pi_{0,1} \omega=$ $\Pi_{0,1} \omega_{1}=\eta$. Предположим, что $\omega_{1}=\rho(f)(\omega)$, где $f \in F_{E}$. Используя (3.5), видим, что $\omega_{1}^{\prime}=\rho\left(f^{\prime}\right)\left(\omega^{\prime}\right)$, где $f^{\prime} \in F_{E^{\prime}}$ и $f_{i}=n_{i} f_{i}^{\prime} n_{i}^{-1}$. Согласно предложению $3.4 \omega^{\prime}$ и $\omega_{1}^{\prime}$ - формы типа $(1,0)$ и потому $\Pi_{0,1} \delta_{0}\left(f^{\prime}\right)=0$. Ввиду следствия леммы $3.1 \delta_{0}\left(f^{\prime}\right)=0$. Тогда $\Pi_{1,0} \delta_{0}\left(f_{i}\right)=0$, откуда $\delta_{0}\left(f_{i}\right)=0$ в силу следствия 4 предложения 2.1. Таким образом, $\omega_{1}=\operatorname{Ad} f(\omega)$, где $f$ - плоское сечение расслоения $E$.

3.3. Сохраняя введенные выше обозначения, мы опишем теперь множество канонических форм в терминах комплекса $\left(A_{\mathfrak{e}}, d\right)$.

Теорема 3.2. Любую каноническую форму $\omega \in Z^{1}\left(R_{E}\right)$ можно записать в виде $\omega=\psi+\partial h$, где $h \in A_{\mathfrak{e}}^{0} u \psi \in A_{\mathfrak{e}}^{1}$ - однозначно определенная гармоническая форма. При этом форма $[\psi, \psi]$ когомологична нулю в $\left(A_{\mathfrak{e}}, d\right)$.

Обратно, пусть $\psi \in A_{\mathfrak{e}}-$ такая гармоническая форма, что $\Pi_{0,1} \psi \in A_{\mathfrak{e}_{\mathfrak{n}}}$ $u[\psi, \psi]$ когомологична нулю в $\left(A_{\mathfrak{e}}, d\right)$. Тогда $\delta_{1}\left(\Pi_{1,0} \psi\right)=\delta_{1}\left(\Pi_{0,1} \psi\right)=0 u$ существует единственная форма $\omega \in Z^{1}\left(R_{E}\right)$ такая, что $\omega=\psi+\partial h$, где $h \in A_{\mathfrak{e}}^{0}$. Форма $\omega$ является канонической.

ДокАЗАТЕЛЬСТво. Пусть $\omega$ - каноническая форма. Можно считать, что $\omega=$ $\alpha+\eta$, где $\alpha=\Pi_{0,1} \omega$. Согласно п. (iii) предложения 3.4 имеем $\partial \alpha=0$. В силу предложения $2.1 \alpha=\varphi+\partial h$, где $h \in A_{\mathfrak{e}}^{0}$ и $\varphi \in A_{\mathfrak{e}}^{1,0}$ гармоническая, т.е. голоморффная форма. Поскольку $\eta$ гармонична в силу предложения 3.2 , форма $\psi=\varphi+\eta$ также гармонична, причем $\omega=\psi+\partial h$. Согласно разложению Ходжа форма $\varphi$ определена однозначно. Очевидно,

$$
\begin{aligned}
{[\psi, \psi] } & =[\omega-\partial h, \omega-\partial h]=[\omega, \omega]+[\partial h, \partial h-2 \omega] \\
& =2 d \omega+\partial[h, \partial h-2 \omega]=\partial(-2 \bar{\partial} h+[h, \partial h-2 \omega]) .
\end{aligned}
$$

Так как $d[\psi, \psi]=0$, то из следствия 3 предложения 2.1 вытекает, что $[\psi, \psi]$ когомологична нулю.

Обратно, допустим, что мы имеем гармоническую форму $\psi \in A_{\mathfrak{e}}$ такую, что $\Pi_{0,1} \psi \in A_{\mathfrak{e}_{\mathfrak{n}}}$ и $[\psi, \psi]=d \lambda$, где $\lambda \in A_{\mathfrak{e}}^{1}$. Обозначим $\varphi=\Pi_{1,0} \psi, \theta=\Pi_{0,1} \psi$. Очевидно,

$$
\begin{aligned}
{[\varphi, \varphi] } & =\partial \Pi_{1,0} \lambda, \\
{[\theta, \theta] } & =\bar{\partial} \Pi_{0,1} \lambda, \\
2[\theta, \varphi] & =\bar{\partial} \Pi_{1,0} \lambda+\partial \Pi_{0,1} \lambda .
\end{aligned}
$$

Поскольку $[\varphi, \varphi]$ голоморфна, из предложения 2.1 и (3.6) следует, что $[\varphi, \varphi]=$ $\partial \Pi_{1,0} \lambda=0$. Аналогично, из (3.7) вытекает, что $[\theta, \theta]=\bar{\partial} \Pi_{0,1} \lambda=0$. В частности, 
$\delta_{1}(\varphi)=\delta_{1}(\theta)=0$. Мы видим также, что $d \bar{\partial} \Pi_{1,0} \lambda=d \partial \Pi_{0,1} \lambda=0$. Применяя следствие 3 предложения 2.1, выводим из (3.8), что

$$
[\theta, \varphi]=\partial \bar{\partial} g
$$

для некоторого $g \in A_{\mathfrak{e}}^{0}$.

В частности, мы видим, что $\theta$ обладает свойствами формы $\eta$ из предложения 3.2 . Мы можем положить $\theta=\eta$ и использовать отображение $r_{\beta}^{-1} \circ r_{\gamma}: \psi \mapsto \psi^{\prime}$, описываемое формулами (3.5).

Применяя (3.5) к $\varphi$ и используя (1.3) и (3.9), получаем

$$
\operatorname{Ad} n_{i}\left(d \varphi_{i}^{\prime}\right)=-\left[\eta_{i}, \varphi_{i}-\eta_{i}\right]=-\partial \bar{\partial} g_{i}
$$

откуда

$$
\begin{aligned}
\partial \varphi^{\prime} & =0 \\
\bar{\partial} \varphi_{i} & =-\partial\left(\operatorname{Ad} n_{i}^{-1}\left(\bar{\partial} g_{i}\right)\right),
\end{aligned}
$$

где $\left(\operatorname{Ad} n_{i}^{-1}\left(\bar{\partial} g_{i}\right)\right) \in A_{\mathfrak{e}^{\prime}}^{0}$.

Будем теперь искать нужную нам форму $\omega$. Запишем $\omega=\psi+\partial h=\alpha+\eta$, где $\alpha=\varphi+\partial h$ и $h \in A_{\mathfrak{e}}^{0}$ нужно выбрать так, чтобы $\delta_{1}(\omega)=0$. Как и в п. (i) предложения 3.4 , имеем $\omega_{i}^{\prime}=\operatorname{Ad} n_{i}^{-1}\left(\alpha_{i}\right)$. Так как $\partial \alpha=0$, то из (1.3) вытекает, что $\partial \omega^{\prime}=0$. Сечение $h$ можно выбрать так, чтобы $\bar{\partial} \omega^{\prime}=0$. Действительно,

$$
\omega_{i}^{\prime}=\operatorname{Ad} n_{i}^{-1}\left(\varphi_{i}\right)+\operatorname{Ad} n_{i}^{-1}(\partial h),
$$

где в силу $(3.5)$

$$
\operatorname{Ad} n_{i}^{-1}\left(\varphi_{i}\right)=\varphi_{i}^{\prime}+\operatorname{Ad} n_{i}^{-1}\left(\eta_{i}\right) .
$$

Заметим, что $d\left(\operatorname{Ad} n_{i}^{-1}\left(\eta_{i}\right)\right)=0$. Действительно, из (3.5) следует, что $\psi^{\prime}=$ $-\operatorname{Ad} n_{i}^{-1}\left(\eta_{i}\right)$ соответствует форме $\psi=0$. Значит, $\delta_{1}\left(\psi^{\prime}\right)=0$. Но $\left[\psi^{\prime}, \psi^{\prime}\right]=0$ в силу предложения 3.2 , так что $d \psi^{\prime}=0$. Отсюда следует, что $d\left(\operatorname{Ad} n_{i}^{-1}\left(\varphi_{i}\right)\right)=d \varphi_{i}^{\prime}$. Из (3.10) видно, что $\partial\left(\operatorname{Ad} n_{i}^{-1}\left(\varphi_{i}\right)\right)=0$ и что форма $\bar{\partial}\left(\operatorname{Ad} n_{i}^{-1}\left(\varphi_{i}\right)\right)$ является $\partial$-точной. В силу п. (iii) леммы 3.1 сушествует такое $h^{\prime} \in A_{\mathfrak{e}^{\prime}}^{0}$, что форма

$$
\operatorname{Ad} n_{i}^{-1}\left(\varphi_{i}\right)+\partial h_{i}^{\prime}=\operatorname{Ad} n_{i}^{-1}\left(\varphi_{i}+\partial\left(\operatorname{Ad} n_{i}\left(h_{i}^{\prime}\right)\right)\right) .
$$

голоморфна. Таким образом, сечение $h=\left(h_{i}\right)$, где $h_{i}=\operatorname{Ad} n_{i}\left(h_{i}^{\prime}\right)$, удовлетворяет нашим условиям.

Мы выбрали $h$ так, чтобы $d \omega^{\prime}=0$. Но тогда

$$
\begin{aligned}
{\left[\omega^{\prime}, \omega^{\prime}\right]_{i} } & =\operatorname{Ad} n_{i}^{-1}\left(\left[\alpha_{i}, \alpha_{i}\right]\right)=\operatorname{Ad} n_{i}^{-1}\left(\left[\varphi_{i}+\partial h_{i}, \varphi_{i}+\partial h_{i}\right]\right) \\
& =\partial\left(\operatorname{Ad} n_{i}^{-1}\left(\left[h_{i}, 2 \varphi_{i}+\partial h_{i}\right]\right)\right) .
\end{aligned}
$$

Поскольку эта форма голоморфна, из п. (iі) леммы 3.1 следует, что $\left[\omega^{\prime}, \omega^{\prime}\right]=0$, откуда $\delta_{1}\left(\omega^{\prime}\right)=0$. Значит, $\delta_{1}(\omega)=0$ и потому $\omega$-каноническая форма.

Предположим теперь, что имеется другая форма $\omega_{1}$, удовлетворяюшая нашим условиям и такая, что $\widetilde{\omega}_{1}=\psi+\partial h^{\prime}$, где $h \in A_{\mathfrak{e}}^{0}$. Тогда из вышесказанного следует, что форма $\partial\left(h_{i}^{\prime}-\left(h_{1}^{\prime}\right)_{i}\right)$, где $\left(h_{1}^{\prime}\right)_{i}=\operatorname{Ad} n_{i}^{-1}\left(\left(h_{1}\right)_{i}\right)$, голоморфна. В силу п. (ii) леммы 3.1 имеем $h^{\prime}=h_{1}^{\prime}$, откуда $\omega=\omega_{1}$. 
3.4. Теперь мы можем сформулировать наш основной результат. Предположим, что $M$ - компактное кэлерово многообразие. Фиксируем $\xi \in H^{0,1}(M, S)$, гармоническую форму $\chi$, лежашую в этом классе, и коцикл Чеха $z=\left(z_{i j}\right)$, представляющий класс $\mu([\gamma]) \in H^{1}(M, K)$, где $\gamma=-\bar{\chi}+\chi \in Z^{1}\left(R_{K}\right)$. Мы хотим описать подмножество

$$
H_{\xi}=\left(\Pi_{0,1}^{*}\right)^{-1}\left(\left(p_{1}^{*}\right)^{-1}(\xi)\right) \subset H_{D R}^{1}(M, G)
$$

(см. (3.1)). Для этого заметим, что $H_{\xi}$ отождествляется с множеством

$$
\widetilde{H}_{\xi}=\left(r_{\gamma}^{*}\right)^{-1}\left(H_{\xi}\right)=\operatorname{Ker}\left(p^{*} \circ \Pi_{0,1}^{*}\right) \subset H_{D R}^{1}(M, E)
$$

(см. (3.2)). Для описания последнего мы используем гармонические 1-формы из комплекса де Рама $\left(A_{\mathfrak{e}}, d\right)$. Напомним, что пространство $\mathbb{H}_{\mathfrak{e}}^{p}$ гармонических $p$-форм изоморфно $H_{D R}^{p}(M, \mathfrak{e}) \simeq H^{p}\left(M, \mathscr{C}_{\mathfrak{e}}\right)$ (см. п. 2.1). Так как $\mathscr{C}_{\mathfrak{e}}$ - пучок алгебр Ли, то в когомологиях этого пучка имеется естественная операция $[\cdot, \cdot]$, соответствующая при изоморфизме де Рама коммутатору е-значных форм. С другой стороны, имеется естественное действие группы плоских сечений $H_{D R}^{0}(M, E) \simeq H^{0}\left(M, \mathscr{C}_{E}\right)$ на $H_{D R}^{p}(M, \mathfrak{e}) \simeq H^{p}\left(M, \mathscr{C}_{\mathfrak{e}}\right)$, которое описывается действием $\mathrm{Ad}$ на e-значных формах и сохраняет указанную операцию. Определим конические алгебраические подмножества $H_{D R}^{1}(M, \mathfrak{e})_{0} \subset H_{D R}^{1}(M, \mathfrak{e})$ и $H^{1}\left(M, \mathscr{C}_{\mathfrak{e}}\right)_{0} \subset H^{1}\left(M, \mathscr{C}_{\mathfrak{e}}\right)$ формулами

$$
\begin{aligned}
H_{D R}^{1}(M, \mathfrak{e})_{0} & =\left\{u \in H_{D R}^{1}(M, \mathfrak{e}): p^{*} \Pi_{0,1}^{*} u=0,[u, u]=0\right\}, \\
H^{1}\left(M, \mathscr{C}_{\mathfrak{e}}\right)_{0} & =\left\{u \in H^{1}\left(M, \mathscr{C}_{\mathfrak{e}}\right): p^{*} \iota_{\mathfrak{e}}^{*}(u)=0,[u, u]=0\right\},
\end{aligned}
$$

где $\iota_{\mathfrak{e}}: \mathscr{C}_{\mathfrak{e}} \rightarrow \mathscr{O}_{\mathfrak{e}}-$ естественное вложение пучков (см. п. 1.3) и $p^{*}: H^{0,1}(M, \mathfrak{e}) \rightarrow$ $H^{0,1}(M, \mathfrak{s}), p^{*}: H^{1}\left(M, \mathscr{O}_{\mathfrak{e}}\right) \rightarrow H^{1}\left(M, \mathscr{O}_{\mathfrak{s}}\right)$ индуцируются естественной проекцией $p: \mathfrak{e} \rightarrow \mathfrak{s}$. Очевидно, они инвариантны относительно $H_{D R}^{0}(M, E)$ и $H^{0}\left(M, \mathscr{C}_{E}\right)$ соответственно. Нам потребуется также соответствующее подмножество пространства $\mathbb{H}_{\mathfrak{e}}^{1}$ гармонических $\mathfrak{e}$-значных 1-форм. В силу следствия 2 предложения $2.1 \mathbb{H}_{\mathfrak{e}}^{1}=\mathbb{H}_{\mathfrak{e}}^{1,0} \oplus \mathbb{H}_{\mathfrak{e}}^{0,1}$, где первое слагаемое - это множество замкнутых $(1,0)$-форм, а второе - замкнутых $(0,1)$-форм. Очевидно, $\mathbb{H}_{\mathfrak{e}}^{1}$ инвариантно относительно $H_{D R}^{0}(M, E)$. Определим следуюшее инвариантное подмножество этого пространства:

$$
\mathbb{H}_{\mathfrak{e} 0}^{1}=\left\{\psi \in \mathbb{H}_{\mathfrak{e}}^{1}: \Pi_{0,1} \psi \in \mathbb{H}_{\mathfrak{e}_{\mathfrak{n}}}^{0,1},[\psi, \psi] d \text {-точна }\right\} .
$$

Заметим, что все эти множества и групшы когомологий с точностью до изоморфизма зависят только от класса $\xi$ и не зависят от выбора коциклов $\chi$ и $z$.

Теорема 3.3. Пусть $M$ - компактное кәлерово многообразие и $G$ - связная разрешимая комплексная алгебрачческая группа, обладающая свойством Ходжа. Тогда

$$
H_{D R}^{1}(M, G)=\bigsqcup_{\xi \in H^{0,1}(M, S)} H_{\xi},
$$

где $H_{\xi}$ задается форммлой (3.11).

Сопоставляя каждому классу когомологий $\tau \in H_{\xi}$ гармоническую часть канонической формы, представляющей класс $\left(r_{\gamma}^{*}\right)^{-1}(\tau) \in \widetilde{H}_{\xi} \subset H_{D R}^{1}(M, E), a$ этой гармонической форме - соответствующий класс когомологий со значениями в $\mathscr{C}_{\mathfrak{e}}$, получаем следующие биекиии:

$H_{\xi} \rightarrow \mathbb{H}_{\mathfrak{e} 0}^{1} / H_{D R}^{0}(M, E) \rightarrow H_{D R}^{1}(M, \mathfrak{e})_{0} / H_{D R}^{0}(M, E) \rightarrow H^{1}\left(M, \mathscr{C}_{\mathfrak{e}}\right)_{0} / H^{0}\left(M, \mathscr{C}_{E}\right)$. 
ДокАЗАтЕльство. Разложение (3.12) очевидно. Ясно также, что

$$
\widetilde{H}_{\xi}=\bigsqcup_{\sigma \in \operatorname{Ker} p^{*}}\left(\Pi_{0,1}^{*}\right)^{-1}(\sigma)
$$

Согласно предложению 3.3 любой класс когомологий из $\left(\Pi_{0,1}^{*}\right)^{-1}(\sigma)$ содержит каноническую форму $\omega$. По теореме $3.2 \omega=\psi+\partial h$, где $\psi$ - гармоническая часть формы $\omega$ и $h \in A_{\mathfrak{e}}^{0}$, причем соответствие $\omega \mapsto \psi$ есть биекция между множеством канонических форм из $\left(\Pi_{0,1}^{*}\right)^{-1}(\sigma)$ и множеством форм $\psi \in \mathbb{H}_{\mathfrak{e} 0}^{1}$, для которых $\Pi_{0,1} \psi \in \sigma$. В силу предложения 3.5 две канонические формы $\omega, \omega_{1}$ лежат в одном и том же классе когомологий тогда и только тогда, когда $\omega_{1}=\operatorname{Ad} a(\omega)$ для некоторого $a \in H_{D R}^{0}(M, E)$. При этом предположении имеем $\omega_{1}=\operatorname{Ad} a(\psi)+$ $\partial(\operatorname{Ad} a(h))$, откуда следует, что $\psi_{1}=\operatorname{Ad} a(\psi)$ есть гармоническая часть формы $\omega_{1}$. Обратно, если $\omega_{1}=\psi_{1}+\partial h_{1}$, где $\psi_{1}=\operatorname{Ad} a(\psi)$ гармонична и $a \in$ $H_{D R}^{0}(M, E)$, то канонические формы $\operatorname{Ad} a(\omega)$ и $\omega_{1}$ имеют одну и ту же гармоническую часть и потому совпадают в силу теоремы 3.2. Таким образом, мы получаем биекцию между $\widetilde{H}_{\xi}$ и $\mathbb{H}_{\mathfrak{e} 0}^{1} / H_{D R}^{0}(M, E)$. Учитьвая коммутативность диаграммы (1.20), мы видим, что соответствия между гармоническими формами, когомологиями де Рама и когомологиями со значениями в $\mathscr{C}_{E}$ приводят к биекциям между $\mathbb{H}_{\mathfrak{e} 0}^{1}, H_{D R}^{1}(M, \mathfrak{e})_{0}$ и $H^{1}\left(M, \mathscr{C}_{\mathfrak{e}}\right)_{0}$ и, значит, между $\mathbb{H}_{\mathfrak{e} 0}^{1} / H_{D R}^{0}(M, E)$, $H_{D R}^{1}(M, \mathfrak{e})_{0} / H_{D R}^{0}(M, E)$ и $H^{1}\left(M, \mathscr{C}_{\mathfrak{e}}\right)_{0} / H^{0}\left(M, \mathscr{C}_{E}\right)$.

В заключение этого пункта сделаем два замечания.

ЗАмЕчАниЕ 3.3. Группа параметров $H^{0,1}(M, S)$ в $(3.12)$ изоморфна компактному комплексному тору $(\operatorname{Pic} M)^{\operatorname{dim} S}$ (см. пример 1.1).

ЗАмЕчАНИЕ 3.4 . Пусть $M$ - компактное кэлерово многообразие, $G$ - комплексная группа Ли, $P$ - голоморфное главное расслоение с базой $M$ и слоем $G$. Известно (см. [3], [4]), что если $P$ задается коциклом $z$ со значениями в максимальной компактной подгруппе $K$ группы $G$ (в частности, $P$ плоско), то малые голоморфные деформации расслоения $P$ плоски и параметризуются Ad $P$-значными гармоническими $(0,1)$-формами $\psi$, удовлетворяющими условию $[\psi, \psi]=0$, где $\operatorname{Ad} P$ - расслоение на алгебры Ли, определенное коциклом $\mathrm{Ad} z$. Условие, наложенное на $z$, равносильно тому, что гомоморфизм $\pi_{1}(M) \rightarrow G$, соответствующий расслоению $P$, принимает значения в $K$. Предположим теперь, что $G$ - линейная алгебраическая группа. Параметризация гомоморфизмов, близких к данному, $\operatorname{Ad} P$-значными гармоническими формами позволяет доказать (см. [1]), что многообразие представлений $\operatorname{Hom}\left(\pi_{1}(M), G\right)$ имеет, самое худшее, квадратичные особенности в точках, отвечающих гомоморфизмам со значениями в $K$. В [2] этот факт был доказан для всех вполне приводимых представлений. Теорему 3.3 можно рассматривать как глобальный результат в том же направлении.

3.5. Этот пункт содержит некоторые дополнения к нашему основному результату.

Заметим, во-первых, что те же самые методы позволяют описать множество $H_{D R}^{1}\left(M, E_{N}\right)$, где $E_{N}$, как и вьше, обозначает унитарное плоское расслоение на групшы со слоем $N$, определяемое некоторым коциклом $z \in Z^{1}(\mathfrak{U}, K)$. Чтобы 
сформулировать результат, введем алгебраические подмножества $H_{D R}^{1}\left(M, \mathfrak{e}_{\mathfrak{n}}\right)_{0} \subset$ $H_{D R}^{1}\left(M, \mathfrak{e}_{\mathfrak{n}}\right), H^{1}\left(M, \mathscr{C}_{\mathfrak{e}_{\mathfrak{n}}}\right)_{0} \subset H^{1}\left(M, \mathscr{C}_{\mathfrak{e}_{\mathfrak{n}}}\right)$ и $\mathbb{H}_{\mathfrak{e}_{\mathfrak{n}} 0}^{1} \subset \mathbb{H}_{\mathfrak{e}_{\mathfrak{n}}}^{1}$ формулами

$$
\begin{aligned}
H_{D R}^{1}\left(M, \mathfrak{e}_{\mathfrak{n}}\right)_{0} & =\left\{u \in H_{D R}^{1}\left(M, \mathfrak{e}_{\mathfrak{n}}\right):[u, u]=0\right\} \\
H^{1}\left(M, \mathscr{C}_{\mathfrak{e}_{\mathfrak{n}}}\right)_{0} & =\left\{u \in H^{1}\left(M, \mathscr{C}_{\mathfrak{e}_{\mathfrak{n}}}\right):[u, u]=0\right\} \\
\mathbb{H}_{\mathfrak{e}_{\mathfrak{n}} 0}^{1} & =\left\{\psi \in \mathbb{H}_{\mathfrak{e}_{\mathfrak{n}}}^{1}:[\psi, \psi] d \text {-точна }\right\}
\end{aligned}
$$

Далее, заметим, что класс $\tau \in H_{D R}^{1}(M, E)$ лежит в $\operatorname{Im} i^{*}=\operatorname{Ker} p^{*}$ тогда и только тогда, когда любая каноническая форма $\omega \in \tau$ удовлетворяет условию $\omega \in A_{\mathfrak{e}_{\mathfrak{n}}}$. Действительно, $\omega=\omega_{\mathfrak{n}}+\omega_{\mathfrak{s}}$, где $\omega_{\mathfrak{n}} \in A_{\mathfrak{e}_{\mathfrak{n}}}^{1}$ и $\omega_{\mathfrak{s}} \in A_{\mathfrak{e}_{\mathfrak{s}}}^{1,0}$. Если $p^{*}(\tau)=\varepsilon$, то $p(\omega)=\omega_{\mathfrak{s}}=\delta_{0}(a)$, где $a \in F_{S}$. Очевидно, $\Pi_{0,1} \delta_{0}(a)=0$. Если $M$ компактно, то отсюда следует, что $\delta_{0}(a)=0$, так что $\omega=\omega_{\mathfrak{n}}$. Рассуждения, использованные при доказательстве теоремы 3.3, приводят к следующему результату.

ТеоремА 3.4. Пусть $M$ - компактное кәлерово многообразие, $G$ - связная разрешимая комплексная линейная алгебрачческая группа, обладающая свойством Ходжа, $N$ - ее унипотентный радикал и $E_{N}$ - унитарное плоское расслоение на группь со слоем $N$, определяемое кочиклом $z \in Z^{1}(\mathfrak{U}, K)$. Если сопоставить каждому классу когомологий $\tau \in H_{D R}^{1}\left(M, E_{N}\right)$ гармоническую часть канонической формы, лежсащей в $i^{*}(\tau)$, а әтой гармонической форме - соответствующий класс когомологий де Рама и класс когомологий со значениями в $\mathscr{C}_{\mathfrak{n}}$, то получатся следующие биекиии:

$$
\begin{aligned}
H_{D R}^{1}\left(M, E_{N}\right) & \rightarrow \mathbb{H}_{\mathfrak{e}_{\mathfrak{n}} 0}^{1} / H_{D R}^{0}\left(M, E_{N}\right) \\
& \rightarrow H_{D R}^{1}\left(M, \mathfrak{e}_{\mathfrak{n}}\right)_{0} / H_{D R}^{0}\left(M, E_{N}\right) \rightarrow H^{1}\left(M, \mathscr{C}_{\mathfrak{e}_{\mathfrak{n}}}\right)_{0} / H^{0}\left(M, \mathscr{C}_{E_{N}}\right) .
\end{aligned}
$$

ЗАмЕчАниЕ 3.5. В частности, в простейшем случае $z=e$ мы получаем описание множества $H_{D R}^{1}(M, N)$ в терминах алгебры когомологий де $\operatorname{Paмa~}^{1}(M, \mathfrak{n})$. Так как группа $N$ стягиваема, то имеется биекция между множествами $H_{D R}^{1}(M, N)$ и $\operatorname{Hom}\left(\pi_{1}(M), N\right) / \operatorname{Int} N$.

Теперь мы дадим обобщение теоремы 3.3 , основанное на том факте, что свойство Ходжа связано только с $(0,1)$-частями наших матричных форм. Это наводит на мысль рассмотреть следующий неабелев коцепной комплекс. Пусть $\widehat{G}-$ произвольная комплексная линейная алгебраическая группа и $G$ - ее связная разрешимая алгебраическая подгруппа. Обозначим через $\hat{\mathfrak{g}} \supset \mathfrak{g}$ соответствуюшие алгебры Ли. Рассмотрим тройку $R_{\widehat{G}, G}=\left\{R_{\widehat{G}, G}^{0}, R_{\widehat{G}, G}^{1}, R_{\widehat{G}, G}^{2}\right\}$, определенную формулой

$$
R_{\widehat{G}, G}^{0}=F_{G}, \quad R_{\widehat{G}, G}^{p}=\left\{\alpha \in A_{\hat{\mathfrak{g}}}^{p}: \Pi_{0, p} \alpha \in A_{\mathfrak{g}}^{0, p}\right\}, \quad p=1,2 .
$$

Легко проверить, что $R_{\widehat{G}, G}$ есть подкомплекс (в смысле [7], [8]) комплекса де Рама $R_{\widehat{G}}$. С другой стороны, $R_{G}$ - подкомплекс в $R_{\widehat{G}, G}$, совпадающий с $R_{\widehat{G}, G}$ в случае $\widehat{G}=G$. Определяются группа 0-когомологий $H^{0}\left(R_{\widehat{G}, G}\right)$ (совпадаюшая с $\left.H^{0}\left(R_{G}\right)\right)$ и множество 1-когомологий $H^{1}\left(R_{\widehat{G}, G}\right)=Z^{1}\left(R_{\widehat{G}, G}\right) / \rho\left(F_{G}\right)$.

Как и в п. $1.3, \Pi_{0,1}$ порождает гомоморфизм множеств с отмеченными точками $\Pi_{0,1}^{*}: H^{1}\left(R_{\widehat{G}, G}\right) \rightarrow H^{0,1}(M, G)$. Предположим, что $M$ - компактное кэлерово многообразие, а $G$ обладает свойством Ходжа. Тогда $\Pi_{0,1}^{*}$ сюръективен. 
Для описания множества $H^{1}\left(R_{\widehat{G}, G}\right)$ мы заменим $R_{\widehat{G}, G}$ подкрученным комплексом. Пусть, как и выше, $G=N \rtimes S$ и $K$ - компактная часть тора $S$. Фиксируем класс $\xi \in H^{0,1}(M, S)$, гармоническую форму $\chi$, лежашую в этом классе, и коцикл Чеха $z=\left(z_{i j}\right)$, представляющий класс $\mu([\gamma]) \in H^{1}(M, K)$, где $\gamma=-\bar{\chi}+\chi \in Z^{1}\left(R_{K}\right)$. Обозначим через $\widehat{E}$ плоское расслоение на группы со слоем $G$, определяемое коциклом Int $z$ со значениями в Aut $\widehat{G}$, и через $\hat{\mathfrak{e}}-$ соответствуюшее расслоение на алгебры Ли. Ясно, что рассмотренные выше плоские расслоения $E$ и е являются подрасслоениями в $\widehat{E}$ и $\hat{\mathfrak{e}}$ соответственно. Тогда можно определить подкомплекс $R_{\widehat{E}, E}$ комплекса $R_{\widehat{E}}$, заданный формулами

$$
R_{\widehat{E}, E}^{0}=F_{E}, \quad R_{\widehat{E}, E}^{p}=\left\{\alpha \in A_{\hat{\mathfrak{e}}}^{p}: \Pi_{0, p} \alpha \in A_{\mathfrak{e}}^{0, p}\right\}, \quad p=1,2 .
$$

Отображение $\Pi_{0,1}$ порождает гомоморфизм $\Pi_{0,1}^{*}: H^{1}\left(R_{\widehat{E}, E}\right) \rightarrow H^{0,1}(M, E)$. При этом имеется следуюшая коммутативная диаграмма:

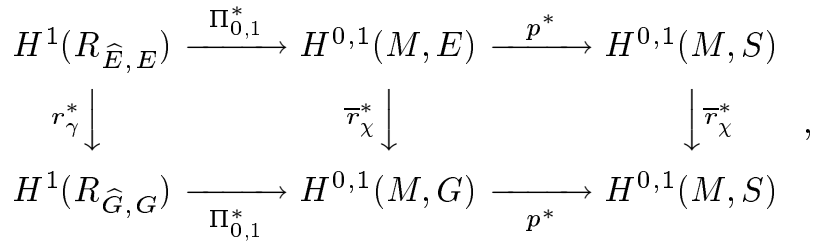

где $r_{\gamma}^{*}$ - биекция, индуцированная отображением $r_{\gamma}: R_{\widehat{E}, E}^{1} \rightarrow R_{\widehat{G}, G}^{1}$.

Определим подмножество

$$
\widehat{H}_{\xi}=\left(\Pi_{0,1}^{*}\right)^{-1}\left(\left(p^{*}\right)^{-1}(\xi)\right) \subset H_{D R}^{1}(M, \widehat{G}) .
$$

Тогда

$$
\left(r_{\gamma}^{*}\right)^{-1}\left(\widehat{H}_{\xi}\right)=\operatorname{Ker}\left(p^{*} \circ \Pi_{0,1}^{*}\right) \subset H_{D R}^{1}(M, \widehat{E}) .
$$

Выберем $\tau \in\left(r_{\gamma}^{*}\right)^{-1}\left(\widehat{H}_{\xi}\right)$. Форма $\omega \in \tau$ называется канонической, если $\Pi_{0,1} \omega \in$ $Z^{1}\left(E_{N}\right)$. Из наших предположений выводится, что каждый такой класс $\tau$ содержит каноническую форму, единственную с точностью до преобразования $\mathrm{Ad} g$, где $g$ - плоское сечение расслоения $E$. Наконец, определим следующее коническое подмножество пространства $\mathbb{H}_{\hat{\mathfrak{e}}}^{1}$ :

$$
\mathbb{H}_{\hat{\mathfrak{e}} 0}^{1}=\left\{\psi \in \mathbb{H}_{\hat{\mathfrak{e}}}^{1}: \Pi_{0,1} \psi \in \mathbb{H}_{\mathfrak{e}_{\mathfrak{n}}}^{0,1},[\psi, \psi] d \text {-точна }\right\} .
$$

Тогда мы имеем следующий результат.

ТЕОРема 3.5. Пусть $M$ - компактное кәлерово многообразие и пусть $G$ обладает свойством Ходжа. Тогда

$$
H^{1}\left(R_{\widehat{G}, G}\right)=\underset{\xi \in H^{0,1}(M, S)}{\bigsqcup} \widehat{H}_{\xi},
$$

где $\widehat{H}_{\xi}$ задается формулой (3.13).

Сопоставляя любому классу когомологий $\tau \in \widehat{H}_{\xi}$ гармоническую часть канонической формы, представляющей класс $\left(r_{\gamma}^{*}\right)^{-1}(\tau)$, получаем следуюшую биекиию:

$$
\widehat{H}_{\xi} \rightarrow \mathbb{H}_{\hat{\mathbf{e}} 0}^{1} / H_{D R}^{0}(M, E) .
$$

Доказательство в основном повторяет доказательство теоремы 3.3 . 


\section{Список литературы}

1. Goldman W. M., Millson J. J. The deformation theory of representations of fundamental groups of compact Kähler manifolds // Publ. Math. IHES. 1988. V. 67. P. 43-96.

2. Simpson C. T. Higgs bundles and local systems // Publ. Math. IHES. 1992. V. 75. P. 5-95.

3. Онищик А.Л. О деформациях расслоенных пространств // Докл. АН СССР. 1965. T. 161. № 1. C. 45-47.

4. Онищик А. Л. О деформациях голоморфных расслоенных пространств // Современные проблемы теории аналитических функций. М.: Наука, 1966. С. 236-239.

5. Brudnyi A. $\bar{\partial}$-equations on compact Kähler manifolds and representations of fundamental groups. Research Thesis. Haifa: Technion, 1995.

6. Brudnyi A. Classification theorem for a class of flat connections and representations of Kähler group // Michigan Math. J. 1999. V. 46. № 3. P. 489-514.

7. Онищик А. Л. Некоторые понятия и применения теории неабелевых когомологий // Труды MMO. 1967. Т. 17. C. 45-88.

8. Onishchik A.L. On non-abelian cochain complexes // Вопросы теории групп и гомологической алгебры. Ярославль: ЯрГУ, 1998. С. 171-197.

9. Гриффитс Ф., Харрис Джс. Принципы алгебраической геометрии. Т. 1. М.: Мир, 1982.

10. Винберг Э. Б., Онищик А. Л. Семинар по группам Ли и алгебраическим группам. М.: Наука, 1988.

11. Хелгасон $C$. Дифференциальная геометрия и симметрические пространства. М.: Мир, 1964 .

Негевский университет им. Бен Гуриона, Израиль

Ярославский государственный университет им. П. Г. Демидова

Поступила в редакцию

E-mail: brudnyi@cs.bgu.ac.il; arkadiy@onishchik.msk.ru

16.02 .2000 